Revue des patrimoines

25 | 2014

Le patrimoine de la Grande Guerre

\title{
La Grande Collecte 1914-1918 aux Archives nationales
}

Isabelle Aristide-Hastir and Elsa Marguin-Hamon

\section{OpenEdition}

1 Journals

\section{Electronic version}

URL: http://journals.openedition.org/insitu/11703

DOI: 10.4000/insitu. 11703

ISSN: $1630-7305$

\section{Publisher}

Ministère de la Culture

\section{Electronic reference}

Isabelle Aristide-Hastir and Elsa Marguin-Hamon, «La Grande Collecte 1914-1918 aux Archives nationales », In Situ [Online], 25 | 2014, Online since 23 December 2014, connection on 25 June 2020. URL : http://journals.openedition.org/insitu/11703; DOI : https://doi.org/10.4000/insitu.11703

This text was automatically generated on 25 June 2020 .

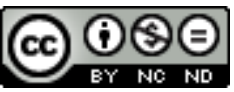

In Situ Revues des patrimoines est mis à disposition selon les termes de la licence Creative Commons Attribution - Pas d'Utilisation Commerciale - Pas de Modification 4.0 International. 


\title{
La Grande Collecte 1914-1918 aux Archives nationales
}

\author{
Isabelle Aristide-Hastir and Elsa Marguin-Hamon
}

1 S'inspirant d'une expérience réalisée à partir de 2011 par la Bibliothèque d'Oxford, une vaste opération de collecte des papiers privés de la Grande Guerre a été menée par l'ensemble des services d'archives nationales et départementales du 9 au 16 novembre 2013. Cette Grande Collecte s'inscrit dans un programme de valorisation à l'échelon européen : Europeana 1914-1918, dont la Bibliothèque nationale a été, entre autres, l'un des pilotes. Ce programme, comme les autres déclinaisons historiques et mémorielles d' Europeana, constitue une occasion unique de mettre en commun des ressources documentaires qui renseignent l'histoire commune à tout le continent, notamment aux grandes nations belligérantes $\mathrm{du} \mathrm{xx}^{\mathrm{e}}$ siècle. Cent ans après, c'est tout un patrimoine testimonial, la mémoire conservée de la guerre dans les familles depuis un siècle, qui est rendu accessible au public via sa numérisation et sa mise en ligne.

\section{Contexte et déroulement de l'opération}

2 Aux Archives nationales, l'opération fut un succès notable, avec plus de 500 particuliers venus déposer, voire donner, lettres, carnets, photos, médailles, dessins - soixantequinze dons d'ensembles documentaires ont été faits à cette occasion. Pour autant, la majorité des détenteurs de fonds ont préféré reprendre leurs documents après l'opération de numérisation. Celle-ci s'est faite en deux temps : les jours de la Grande Collecte elle-même, et postérieurement, l'affluence, la quantité des documents et leur intérêt n'ayant pas permis d'en assurer la numérisation durant les quelques jours impartis à l'opération. De nombreuses familles ont en outre consenti à rapporter pour un don définitif aux Archives nationales à l'issue des quatre ans de commémoration des papiers dont ils souhaitaient pour l'heure assurer la valorisation dans le cadre de manifestations familiales ou locales. Tant il est vrai que le contexte commémoratif et l'intérêt nouveau porté par des institutions comme la Bibliothèque nationale, les Archives de France et les Archives nationales à ce matériau documentaire ont suscité 
chez les propriétaires d'archives privées de la Grande Guerre l'efflorescence d'initiatives nombreuses et variées, dont témoignent les réseaux sociaux, les sites d'histoire locale et les blogs spécialement conçus à cette occasion pour publier transcriptions de souvenirs de guerre, iconographie numérisée, etc.

Ces ensembles patrimoniaux furent longtemps oubliés dans les greniers ou les fonds d'armoire par des descendants pour qui le souvenir du premier conflit s'était trouvé peu à peu relégué au second plan du récit familial. Assurant ouvertement le lien entre histoire personnelle et "grande histoire", ils s'avèrent aujourd'hui un puissant catalyseur mémoriel, permettant au grand public de réinvestir sur un mode sensible le souvenir de la Grande Guerre. C'est pourquoi l'opération de collecte menée par les Archives nationales a porté une attention particulière aux explications et témoignages fournis par les donateurs et les déposants: elles offrent en effet de précieuses informations, à la fois sur l'origine et l'histoire des documents, mais aussi sur les phénomènes d'appropriation et de réappropriation familiale dont ces papiers sont l'objet.

\section{Richesse et diversité des documents}

4 L'ensemble documentaire, résultat d'une collection formée par le hasard et sans critère sélectif a priori, a un aspect de prime abord, hétéroclite; si l'on retrouve des typologies récurrentes (lettres, cartes postales, livrets militaires, carnets de notes, photographies, citations), chaque apport a sa particularité quant à la typologie ou au contenu documentaire et contribue à former une collection d'une très grande richesse, unique en son genre aux Archives nationales,

5 Au-delà même des documents qui sont mis en ligne sur le portail Europeana 1914-1918, les archives collectées témoignent à la fois de l'urgence clairement ressentie par les soldats et leurs familles de rendre compte, pour eux-mêmes et leurs proches, du caractère exceptionnel des événements vécus au quotidien, mais aussi de la diversité des moyens utilisés pour ce faire. Par opposition aux lettres et cartes de soldats, souvent assez brèves et informatives - consignes et censure du courrier obligent - les dessins ou les courriers d'enfants qu'ils reçoivent, mais aussi les poèmes, les croquis pris sur le vif, parfois d'un intérêt documentaire saisissant pour l'histoire des tranchées et des troupes, les cahiers ou carnets couverts d'annotations contemporaines du conflit ou de récits entrepris après la démobilisation sont autant de traces, toutes uniques et précieuses. Elles révèlent l'état d'esprit d'une population qui restera profondément marquée par l'expérience du conflit.

6 Nous en livrons ici un échantillon, aussi représentatif que possible.

\section{La Grande Collecte : florilège}


Figure 1

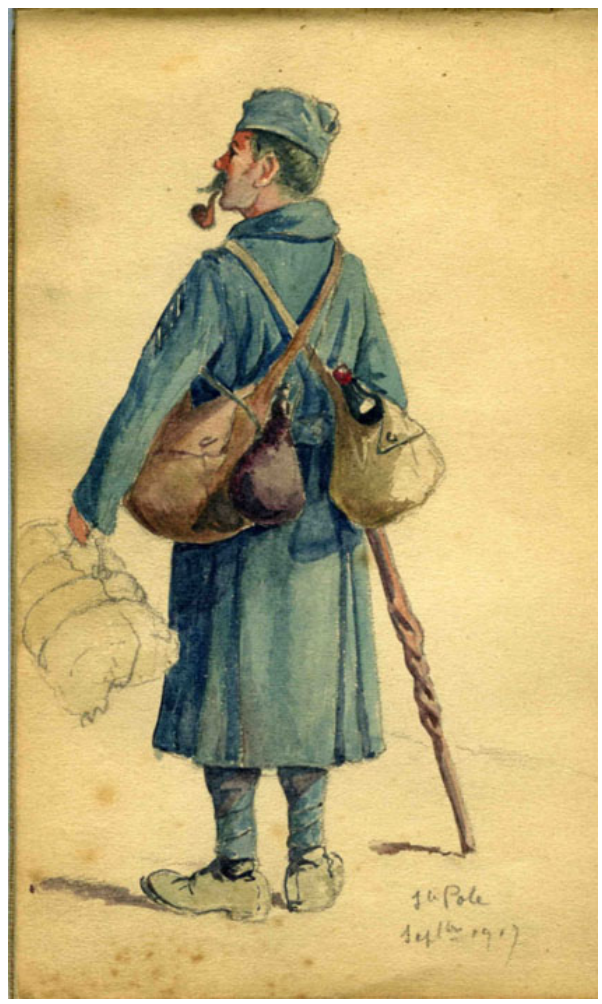

Un poilu en 1977, dessin de Charles Leproux de La Rivière. AN, 700AP/PI_063.

(c) Archives nationales.

7 Les documents privés de la guerre 14-18 apportés par les particuliers lors des journées nationales «Grande Collecte » sont les témoins de la vie quotidienne des soldats, mais aussi de leur famille à l'arrière, à travers des documents officiels militaires, des correspondances, cartes postales et lettres, des carnets de notes, des dessins, des photographies. L'image de la guerre véhiculée par ces supports multiples et fragiles est de deux types: celle que l'on adresse aux familles et aux proches, et que l'on veut, malgré tout, rassurante, et celle vécue par les combattants, dans son immédiateté et sa violence, qui restera, pour certains, enfouie dans les cartons, pour d'autres, partagée avec des camarades de combat, diffusée et publiée dans le but de replacer leur histoire personnelle dans une histoire collective.

8 Parmi les documents de la Grande Collecte, les papiers militaires, ordres d'appel sous les drapeaux, livrets militaire, citations, déclarations de "Mort pour la France", décorations, restent souvent, avec la photographie en uniforme, les seules archives des soldats de la guerre 14-18 précieusement conservées au sein des familles (fig. 1, 2, 3, 4). 
Figure 2

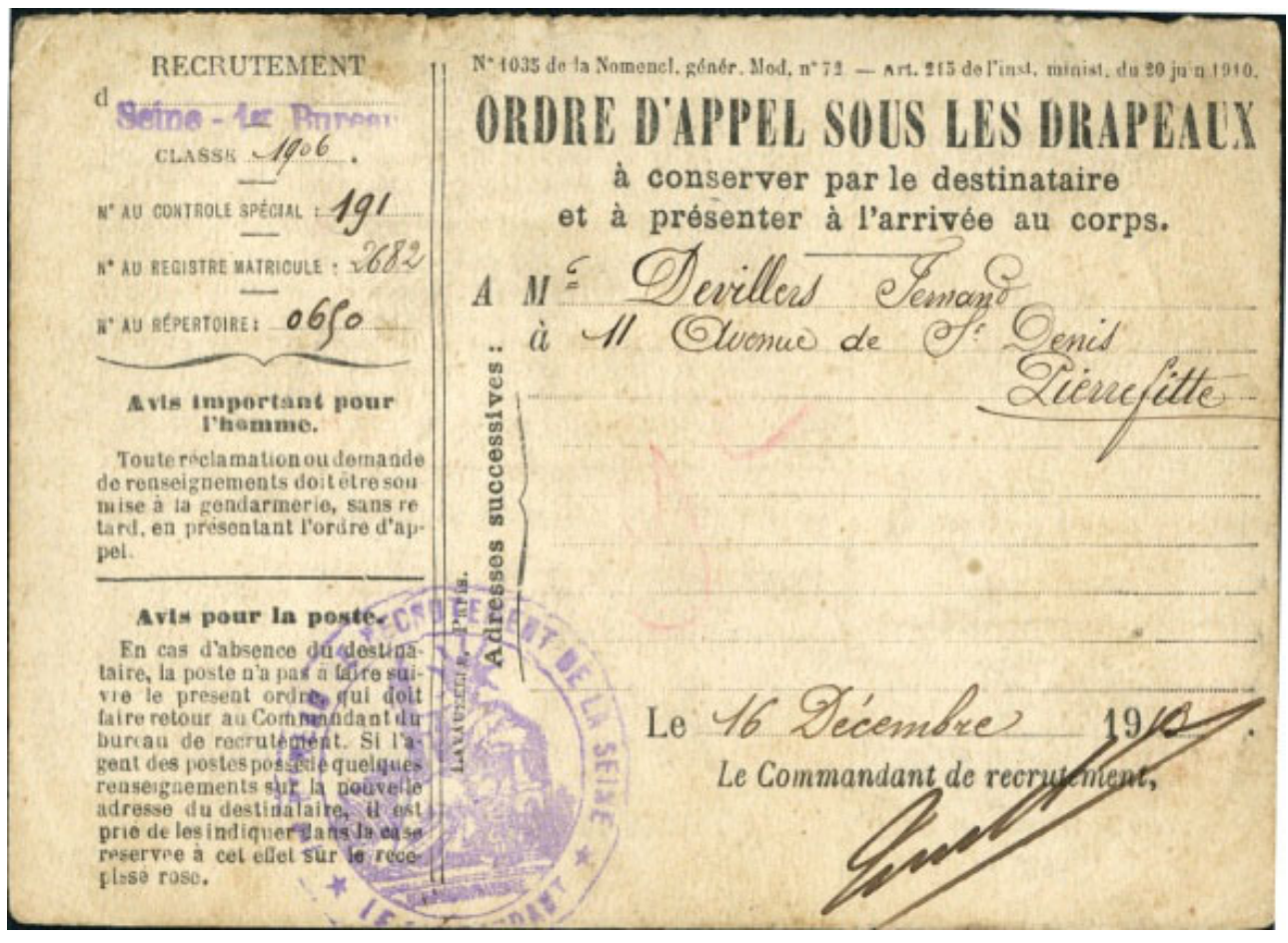

Ordre d'appel sous les drapeaux de Fernand Devillers, de Pierrefitte. (1913). AN, 700AP/PI_002.

(c) Archives nationales.

Figure 3

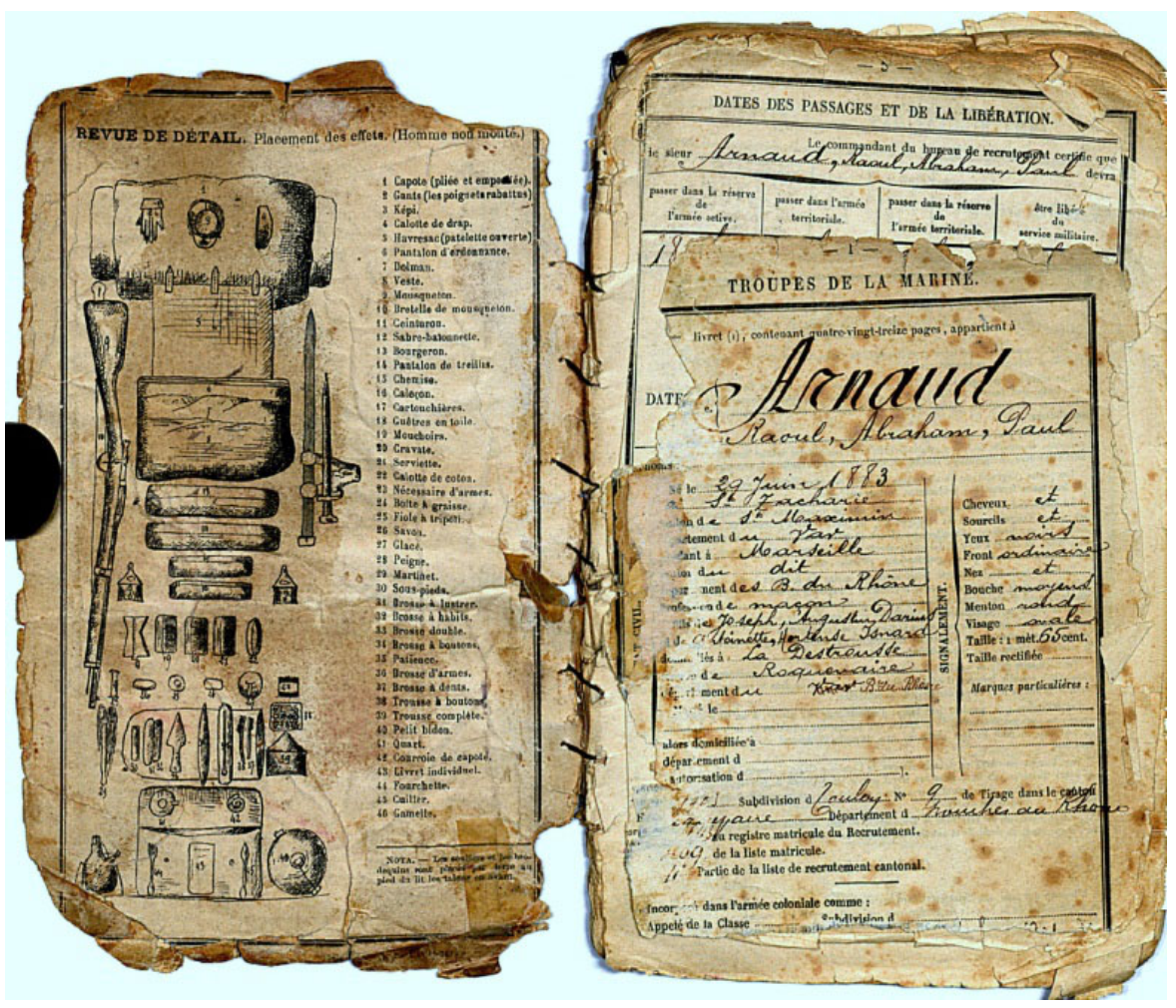

Livret militaire du soldat Raoul Arnauld. AN, 700AP/PA_024.

(c) Archives nationales. 
Figure 4

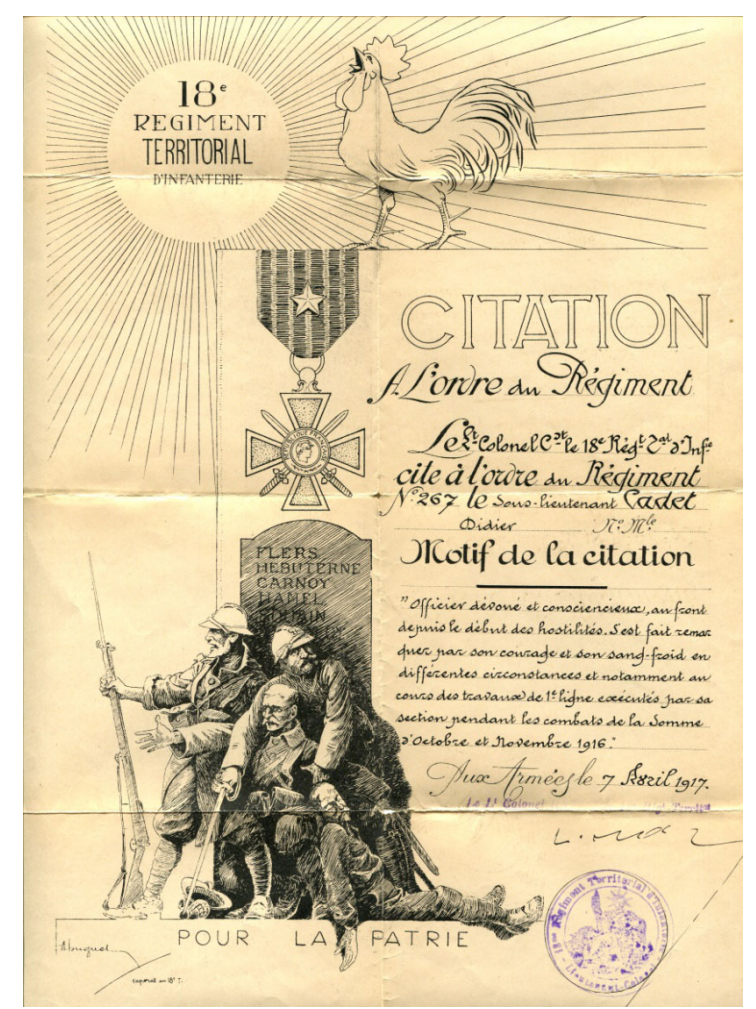

Citation au nom de Didier Cadet, 1917. AN, 700AP/PA_300.

(C) Archives nationales.

9 La photographie du soldat en uniforme ou en groupe au sein de sa compagnie devient vite un document incontournable dans la communication avec les familles; des officines en arrière des lignes de front permettaient au soldat de se faire photographier; les tirages, montés en cartes postales, servaient à la correspondance (fig. 5, 6). 
Figure 5

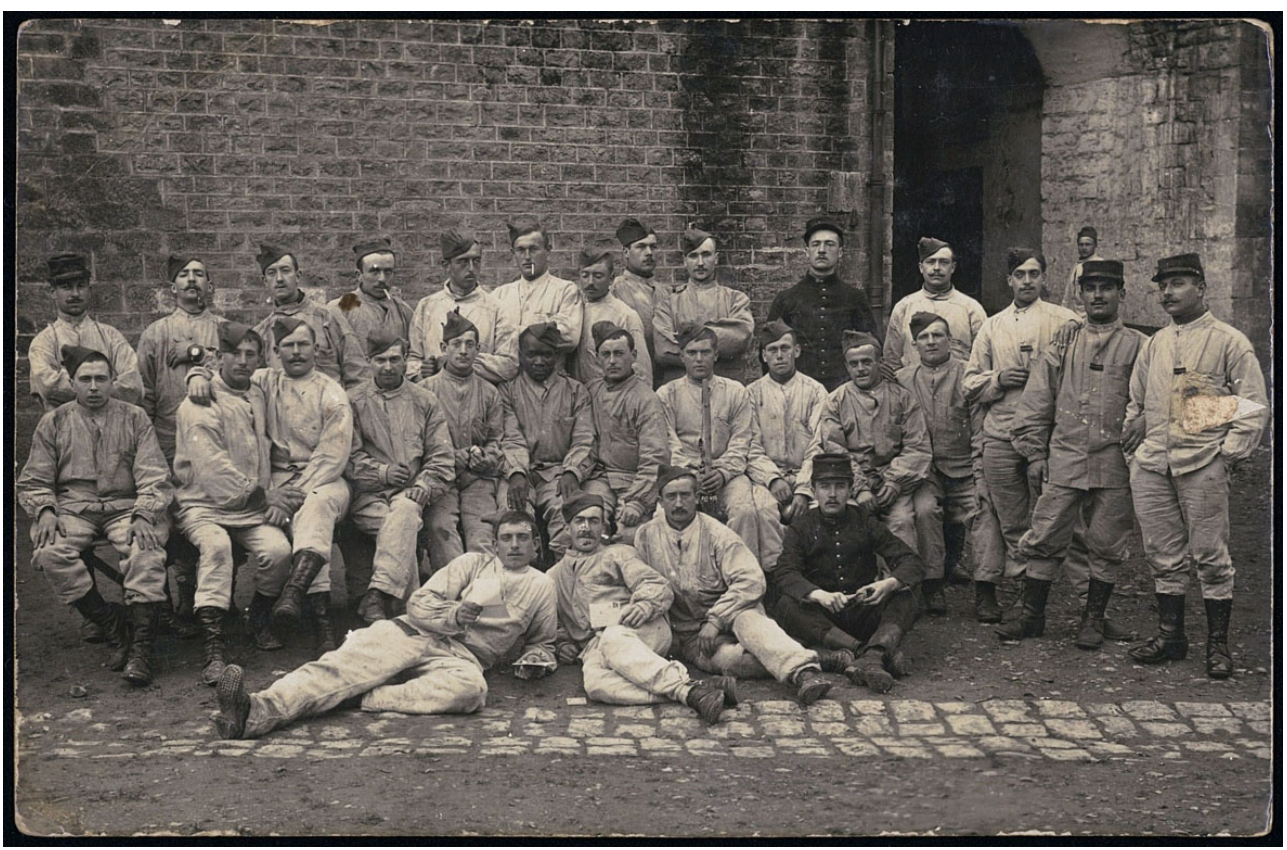

Le soldat César Marchandise et sa compagnie, photographiés près de Sedan « en descendant de la soupe » (1914). AN, 700AP/PI_018.

(c) Archives nationales.

Figure 6

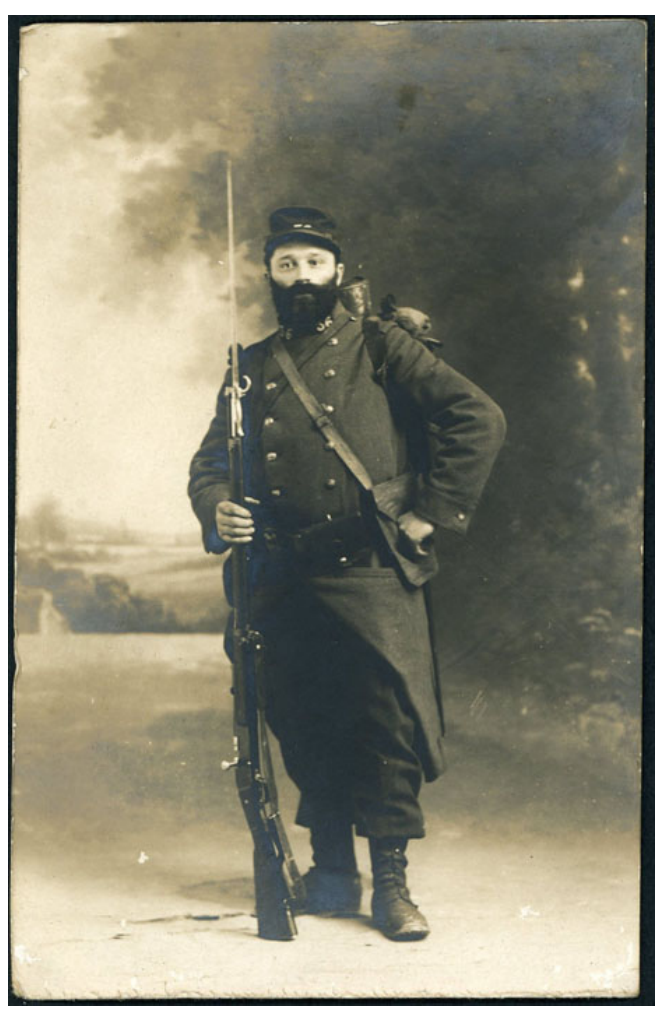

Le soldat Gabriel Otello. AN, 700AP_121.

(C) Archives nationales. 
Comme les lettres, les photographies prises sur le front, envoyées à la famille sous forme de cartes postales, sont des preuves de vie rassurantes. Elles témoignent donc plutôt de la vie quotidienne du soldat : la cantine roulante, le ravitaillement, la toilette au bord de la rivière, la lecture des courriers, le tableau de chasse des rats, les divertissements (jeux de cartes, de boules, théâtre aux armées), et rarement de l'horreur de la vie au front; la mort en est généralement exclue, ou du moins, on ne la représente que sous sa forme acceptable, des tombes soigneusement entretenues...

(fig. $7,8,9,10,11,12$ )

Figure 7

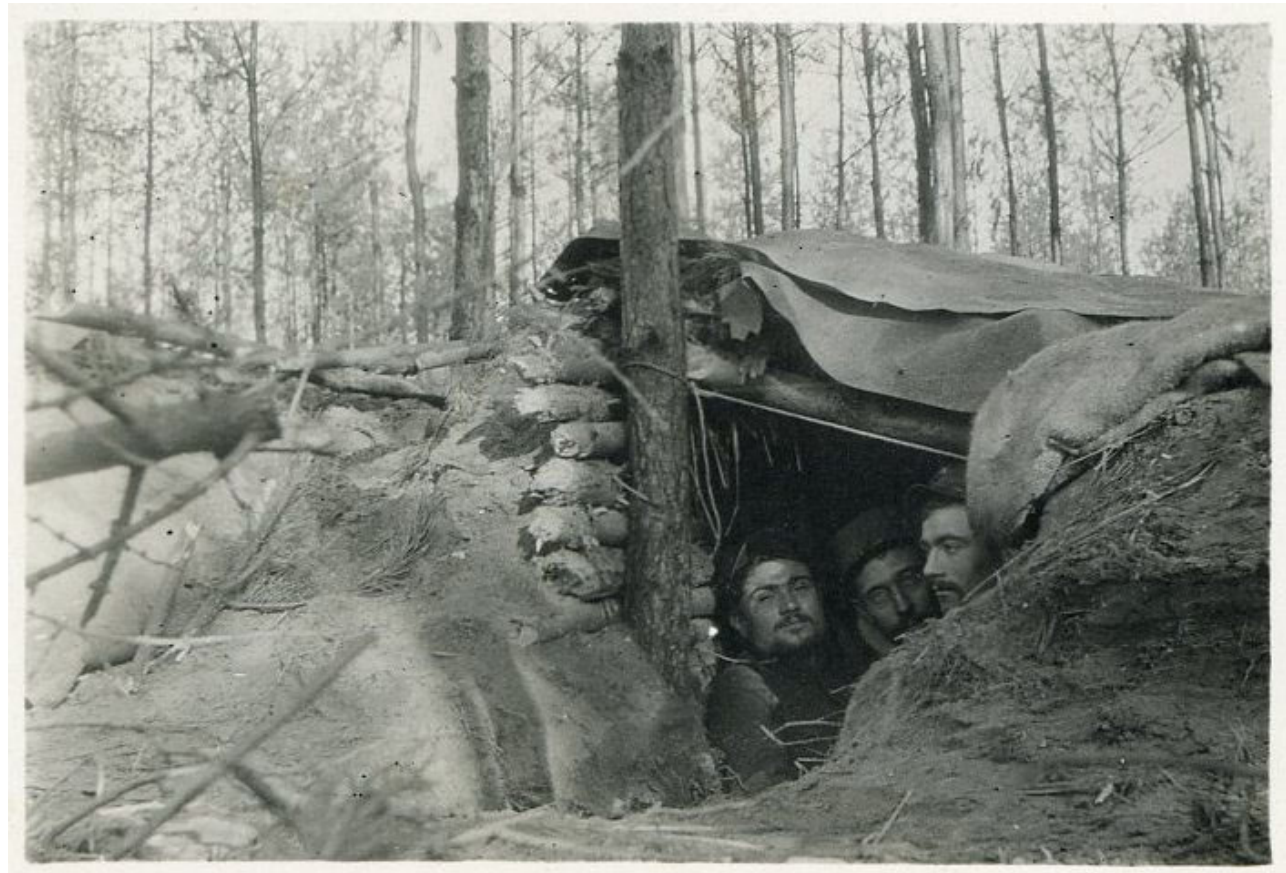

La tranchée, photographie prise par le soldat Jacques Duché. AN, 700AP/PA_296.

(c) Archives nationales. 
Figure 8

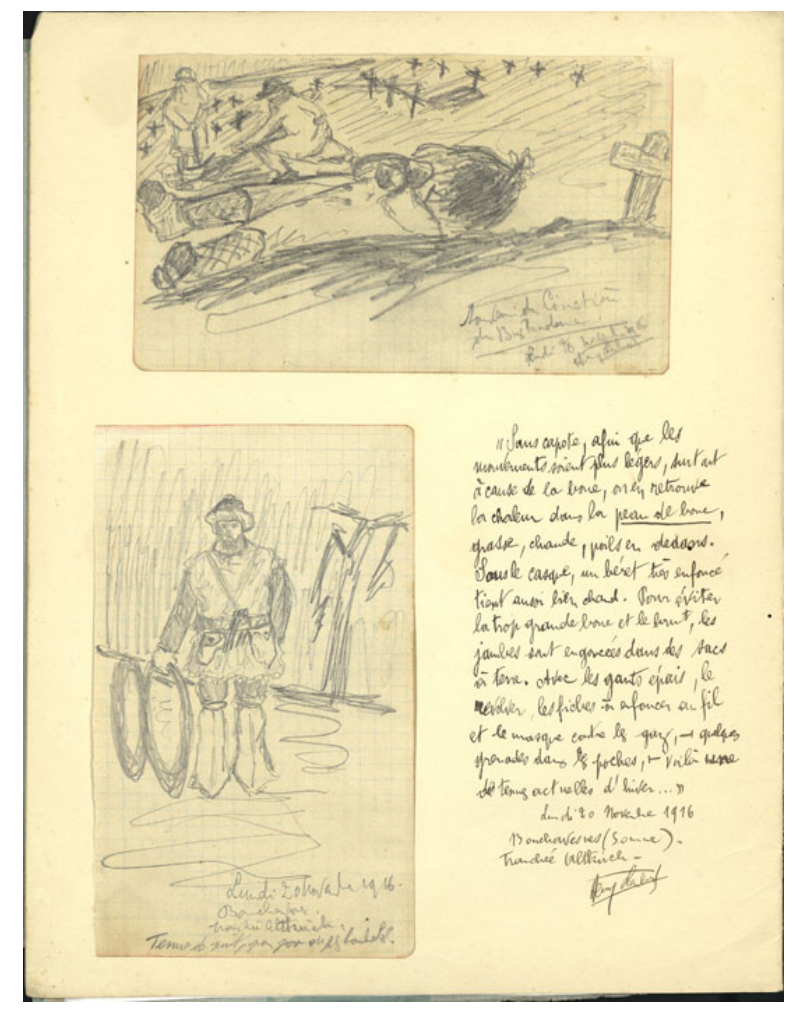

La tranchée, dessin du soldat Henri Lambert. AN, 700AP/PA_324.

(c) Archives nationales. 
Figure 9

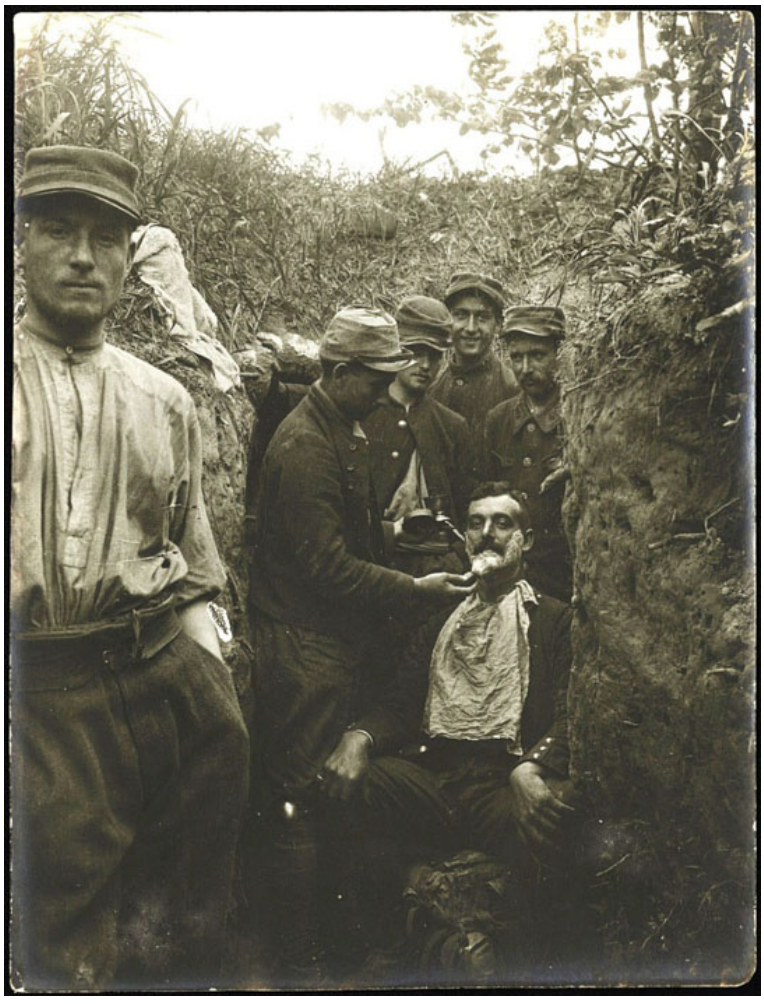

Le barbier. Photographie faite par Paul-Marcel Piquemal. AN, 700AP/PA_159. (c) Archives nationales. 
Figure 10

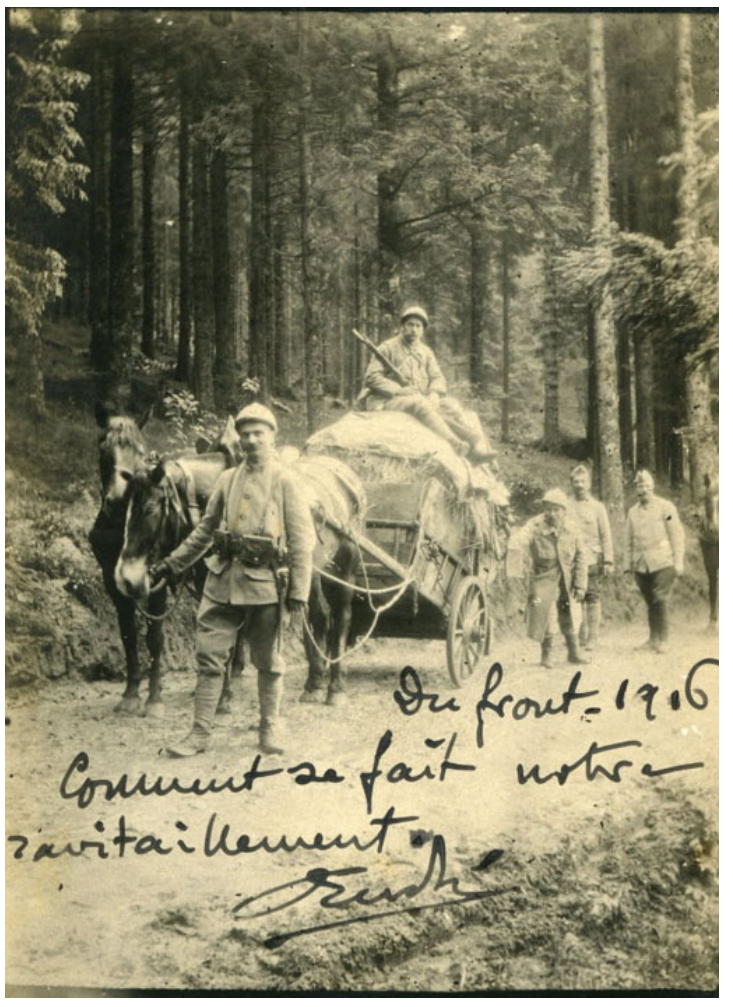

Le ravitaillement, photographie envoyée par le sergent Albert Larivière. AN, 700AP/PI_026. (c) Archives nationales.

Figure 11

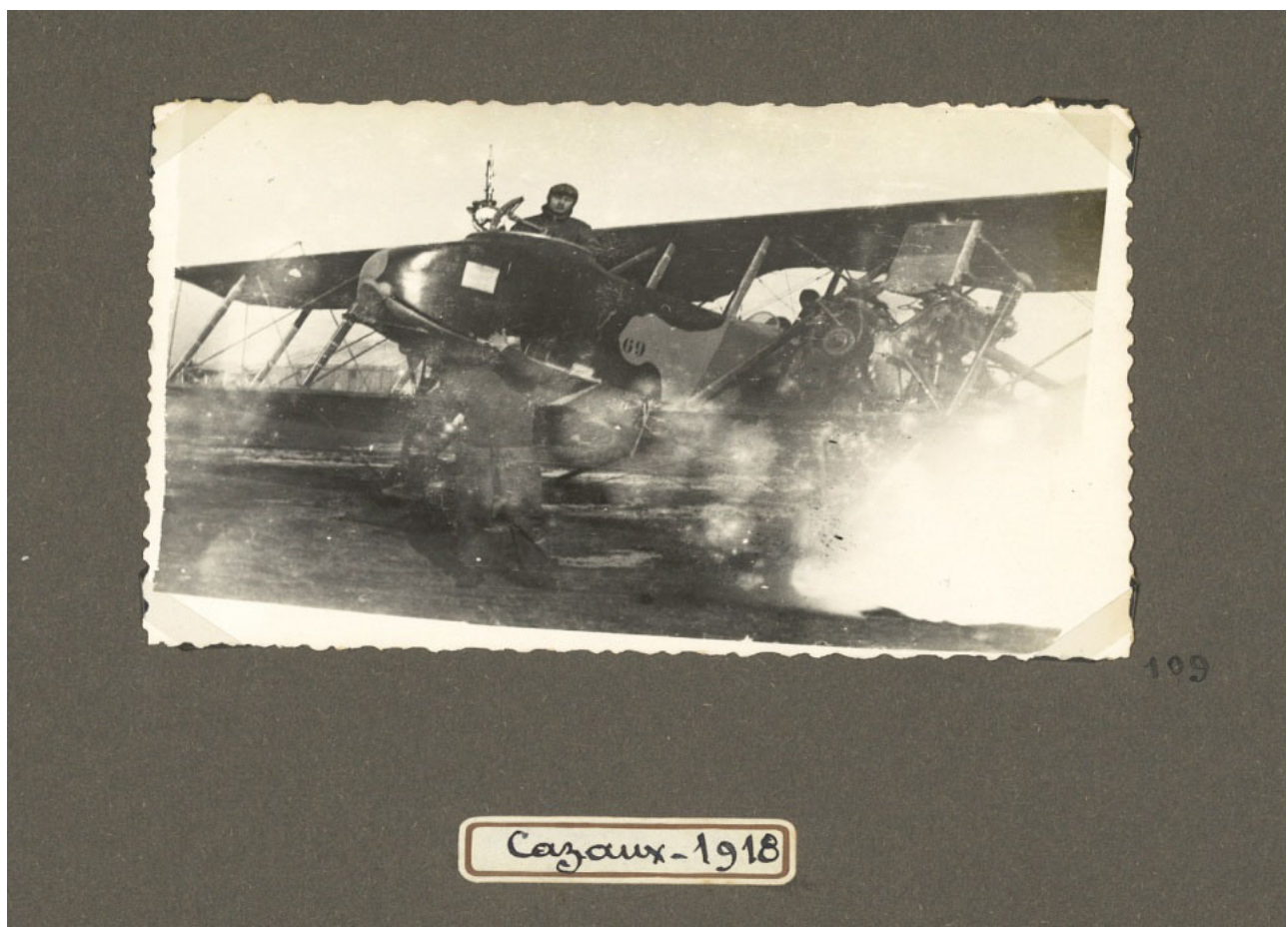

L'aviateur Joseph Martin, qui a commencé la guerre comme simple soldat d'infanterie, AN, 700AP/ PA_347.

(c) Archives nationales. 
Figure 12

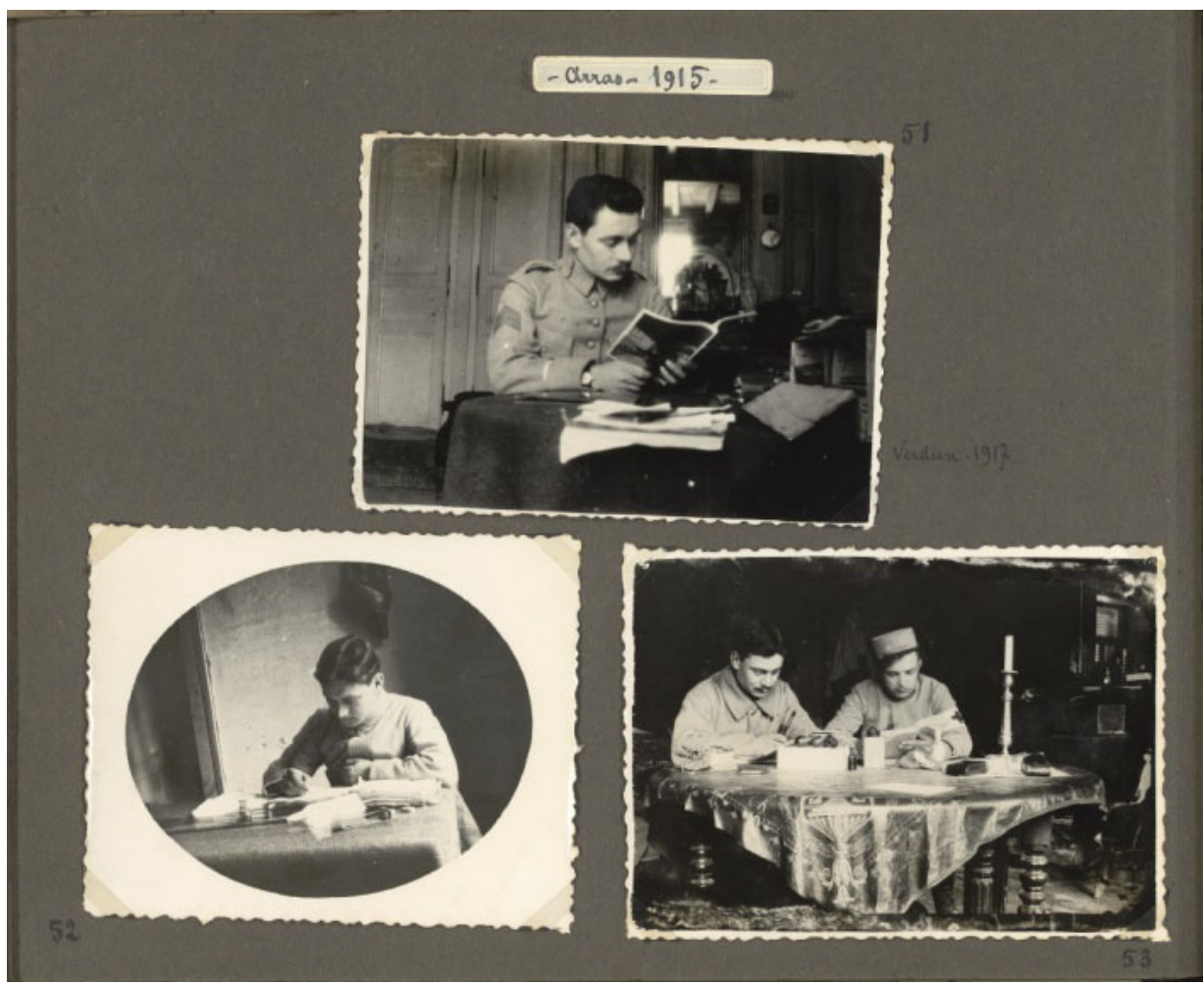

À l'heure du repos : la correspondance, album de Gustave Ardoin. AN/700AP/PA_346. (c) Archives nationales.

11 Mais la guerre de 14-18 voit aussi le développement de l'usage de la photographie et la diffusion de l'appareil photographique portatif; de nombreux soldats ou officiers qui peuvent se permettre cet investissement, réalisent alors, malgré la censure, des clichés réalistes (fig. 13, 14, 15).

Figure 13

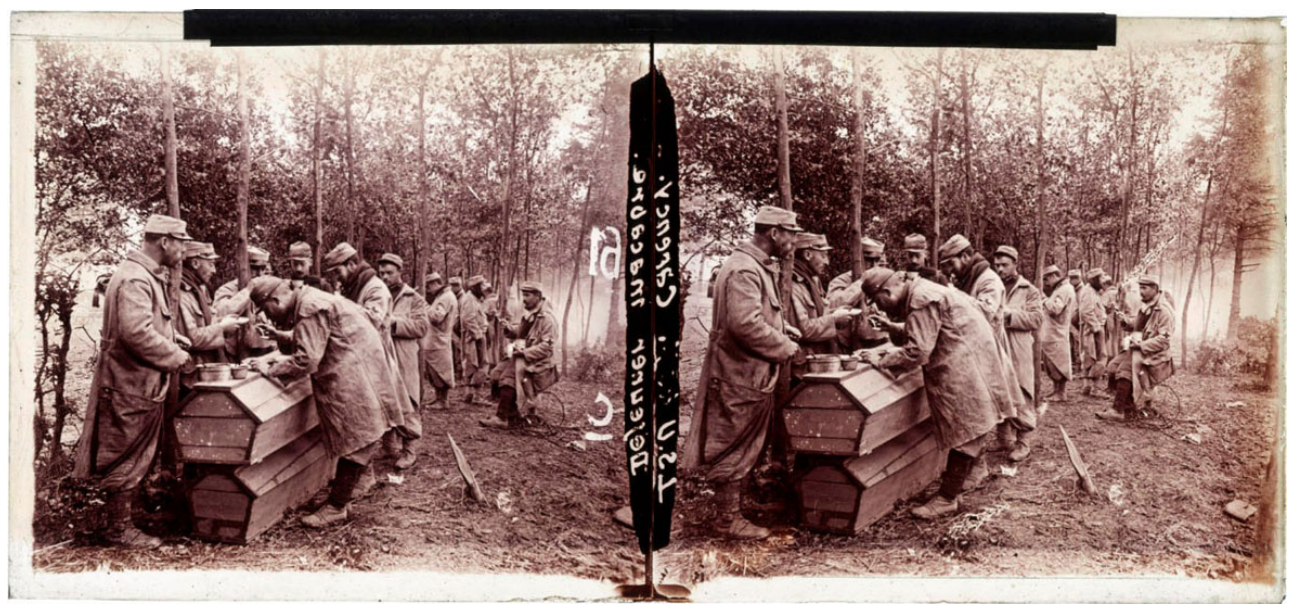

La soupe des poilus, cliché stéréoscopique sur plaque de verre de Nicolas Poutas. AN, 700AP/PA_347. (c) Archives nationales. 
Figure 14

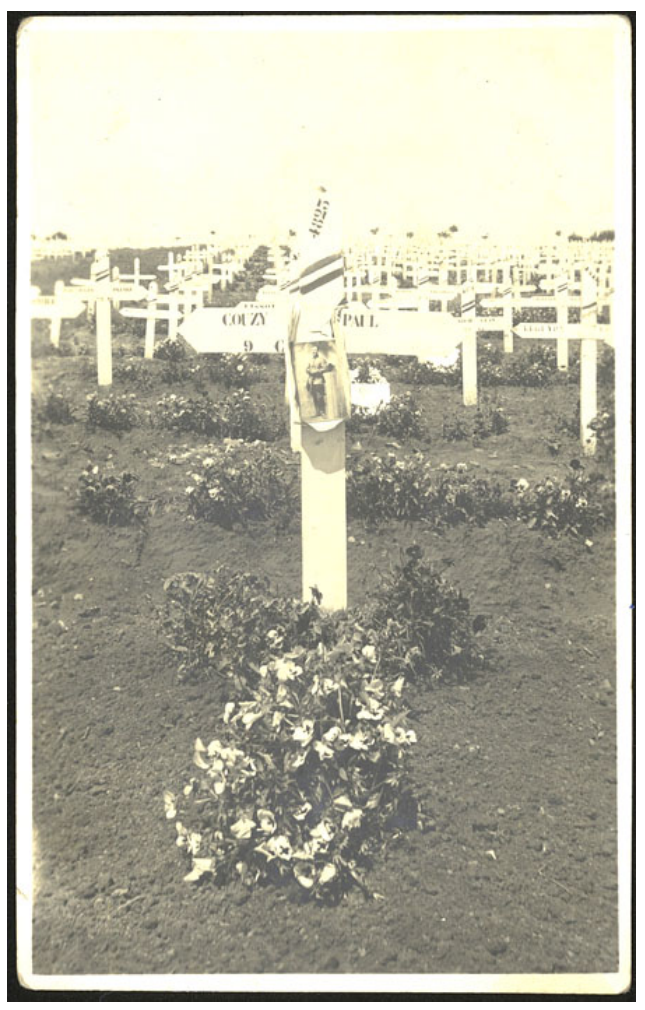

La mort ritualisée : tombe d'un soldat, photographiée par le soldat Lumalé. AN, 700AP/PA_0139. (c) Archives nationales. 
Figure 15

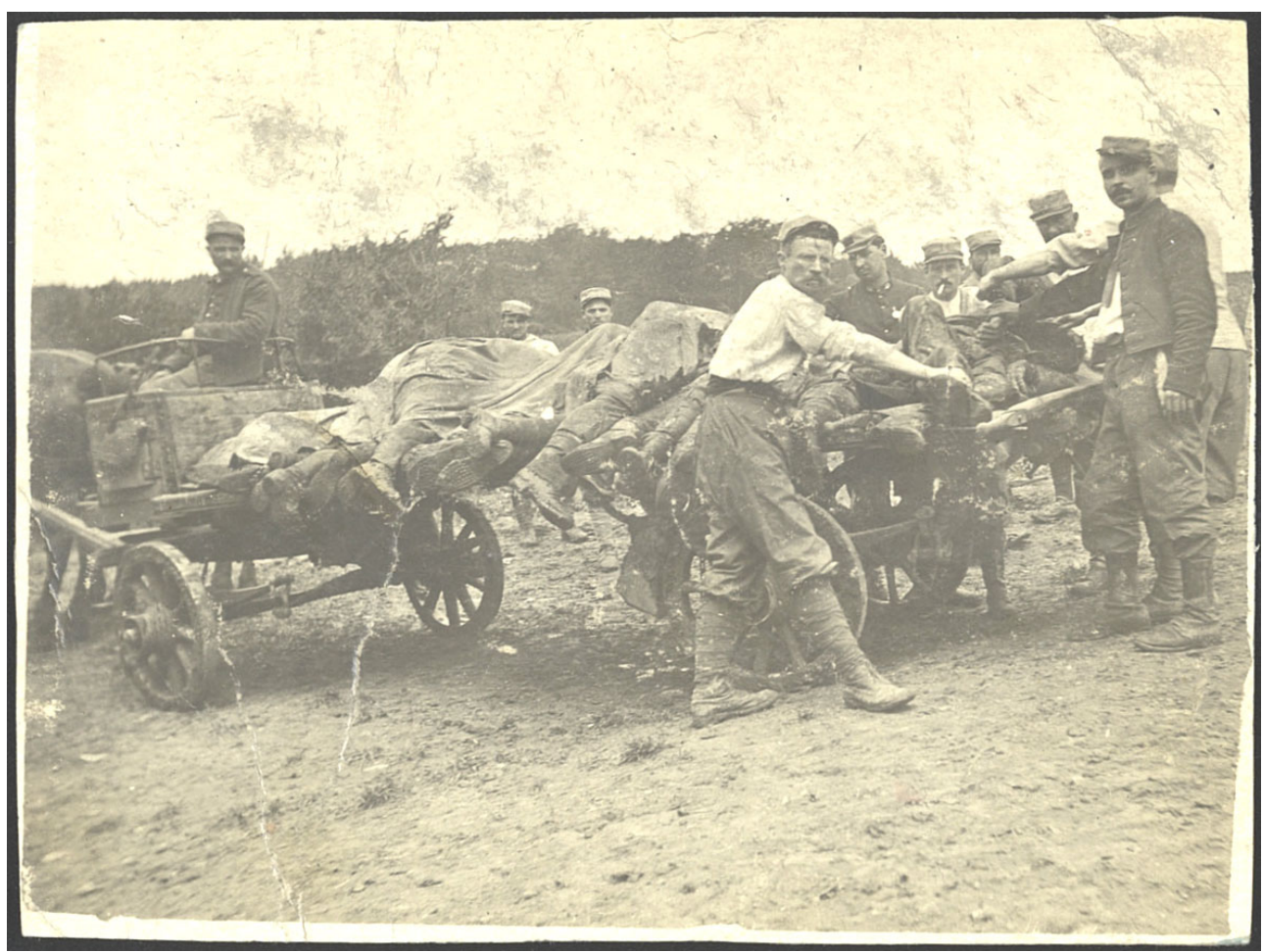

L'enlèvement des morts, photographie du soldat Louis André Guay. AN, 700AP/PA_218.

(c) Archives nationales.

12 Conscients de l'importance des événements dont ils souhaitent garder la mémoire pour eux-mêmes ou pour leur famille, de nombreux soldats ont tenu au jour le jour un carnet de notes manuscrit, qui revêt des formats très divers; souvent griffonné au crayon de bois, il sera, dans certains cas, repris à l'issue du conflit, retravaillé, illustré de dessins, de croquis, de photographies échangées avec les camarades de combat (fig. 16, 17, 18, 19, 20, 21, 22, 23, 24, 25). 
Figure 16

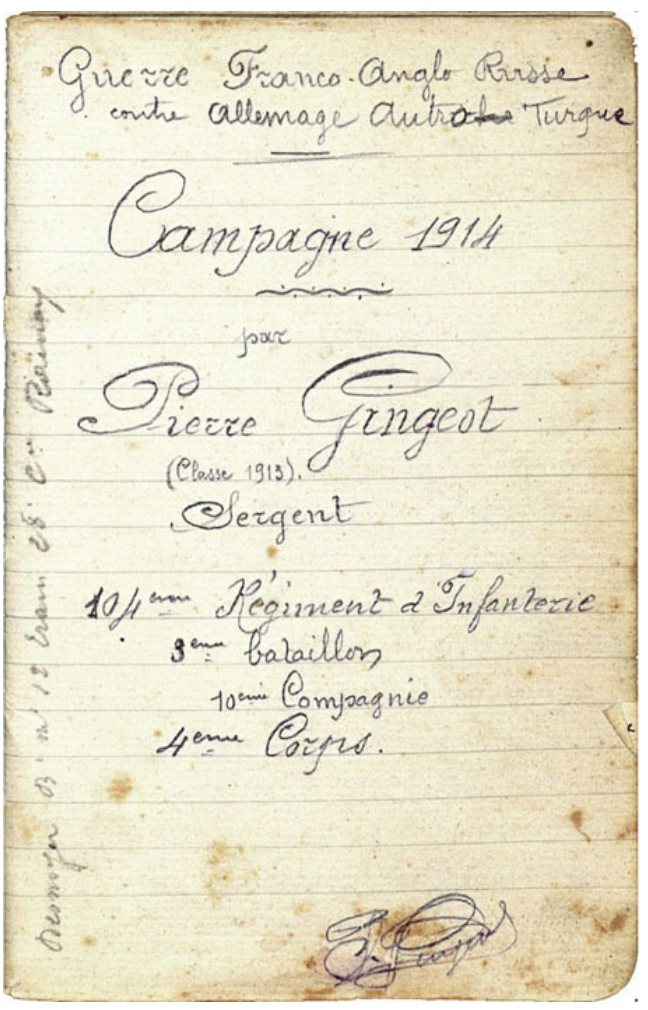

Carnet de notes du sergent Pierre Gingeot. AN, 700AP/PI_032.

(c) Archives nationales. 
Figure 17

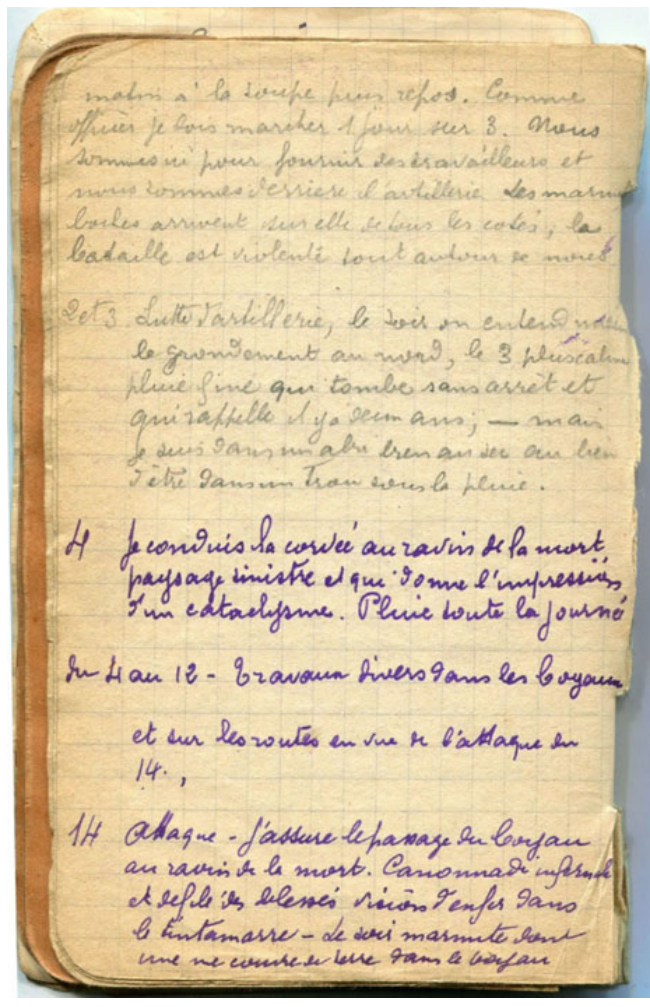

Carnet de notes du sous-lieutenant Didier Cadet, à la page d'octobre 1916. AN, 700AP/PA_300. (c) Archives nationales. 
Figure 18

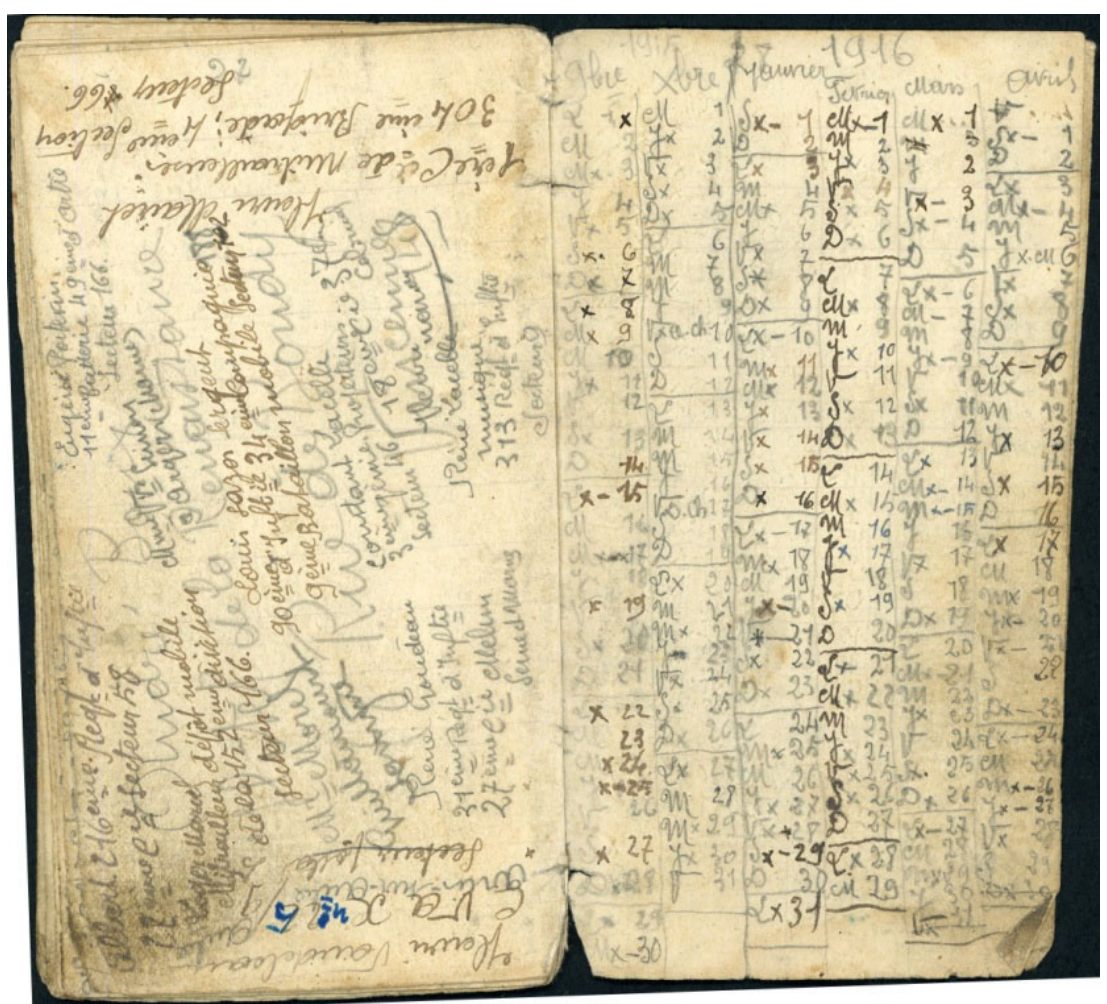

Carnet de notes du soldat Daniel Godu. AN, 700AP/PA_099.

(c) Archives nationales.

Figure 19

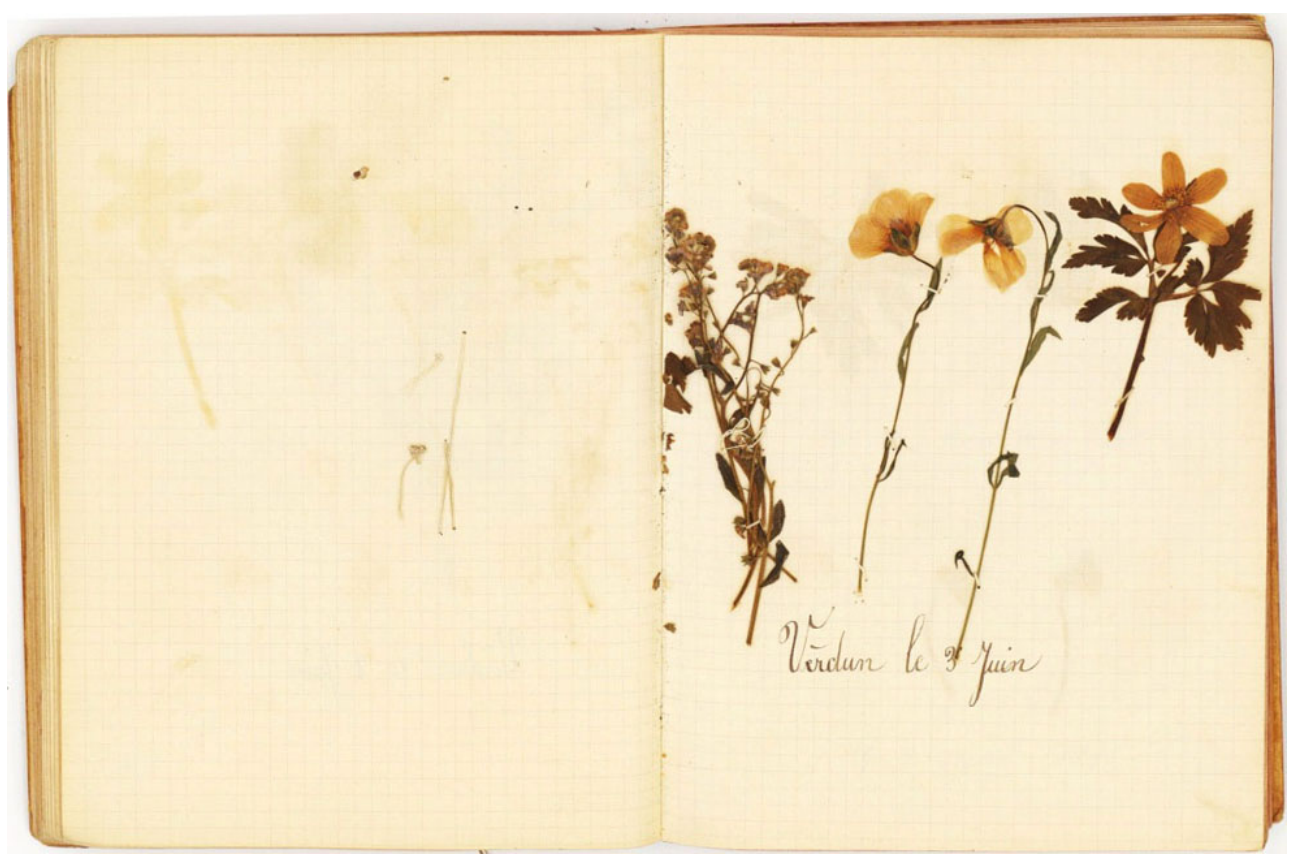

Carnet de notes du soldat Jean-Pierre Luminet, en forme d'herbier, à la page de juin [1916], Verdun. AN 700AP/PA_059.

(C) Archives nationales. 
Figure 20

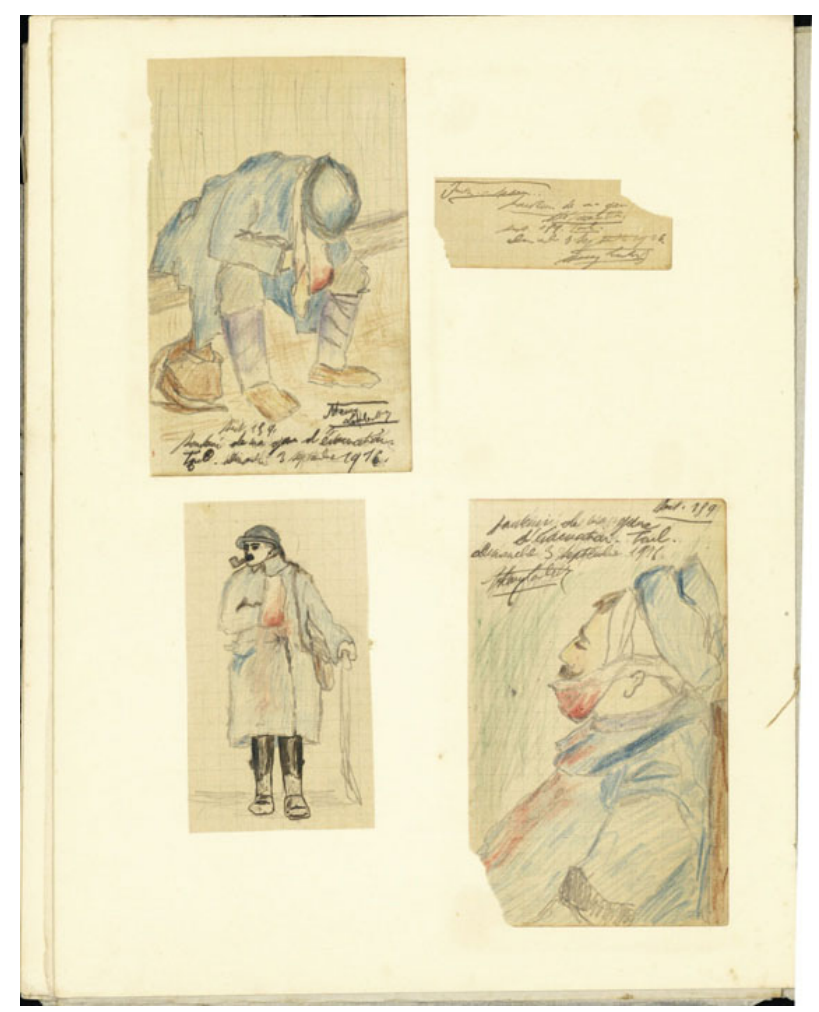

Carnet de notes et de dessins, assemblés après la guerre par le soldat Henri Lambert, intitulé « Quatre ans de bagne ». AN, 700AP/PA_324.

(C) Archives nationales. 
Figure 21

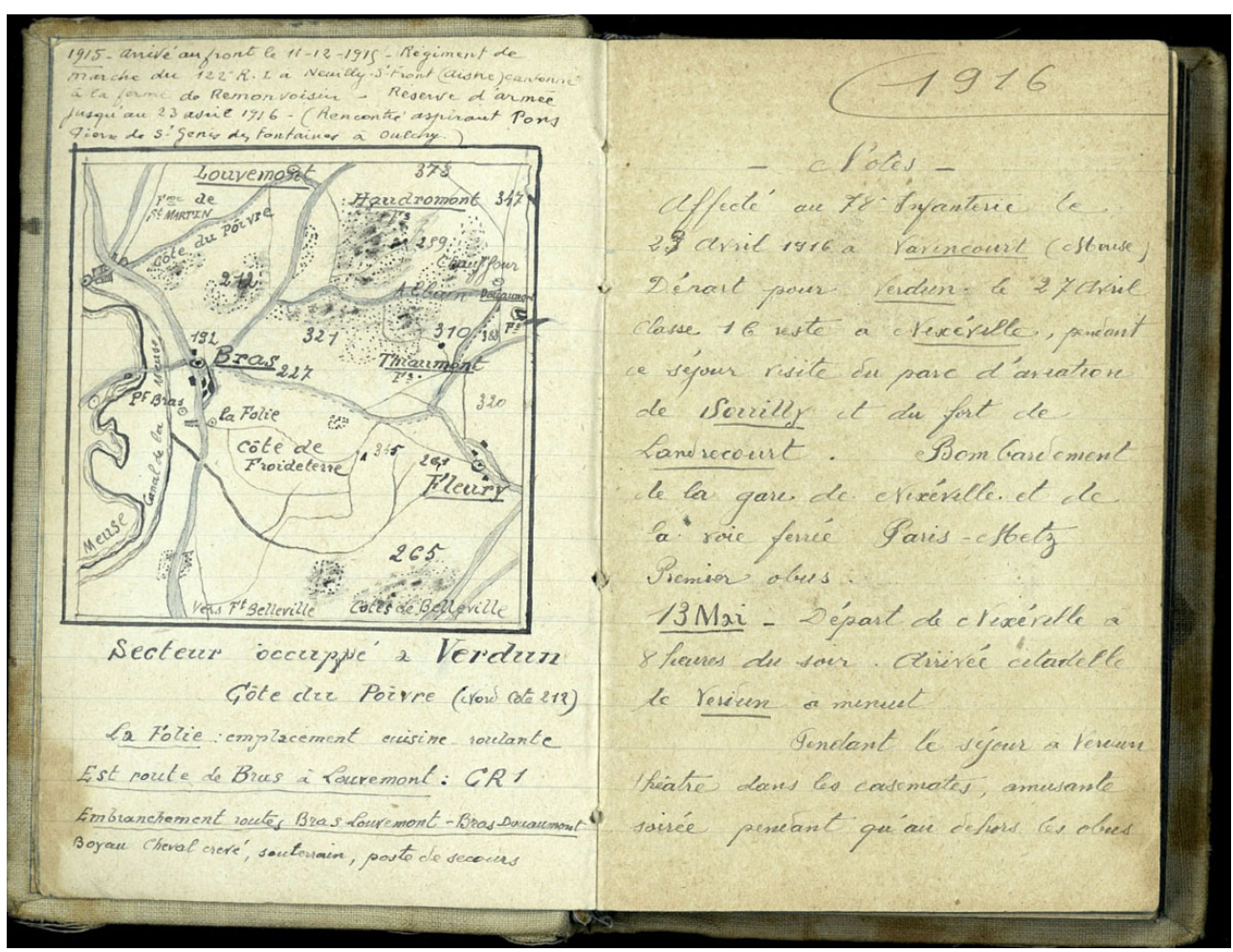

Carnet de Jacques Souquet, retravaillé après guerre. AN, 700AP/PA_017.

(c) Archives nationales.

\section{Figure 22}

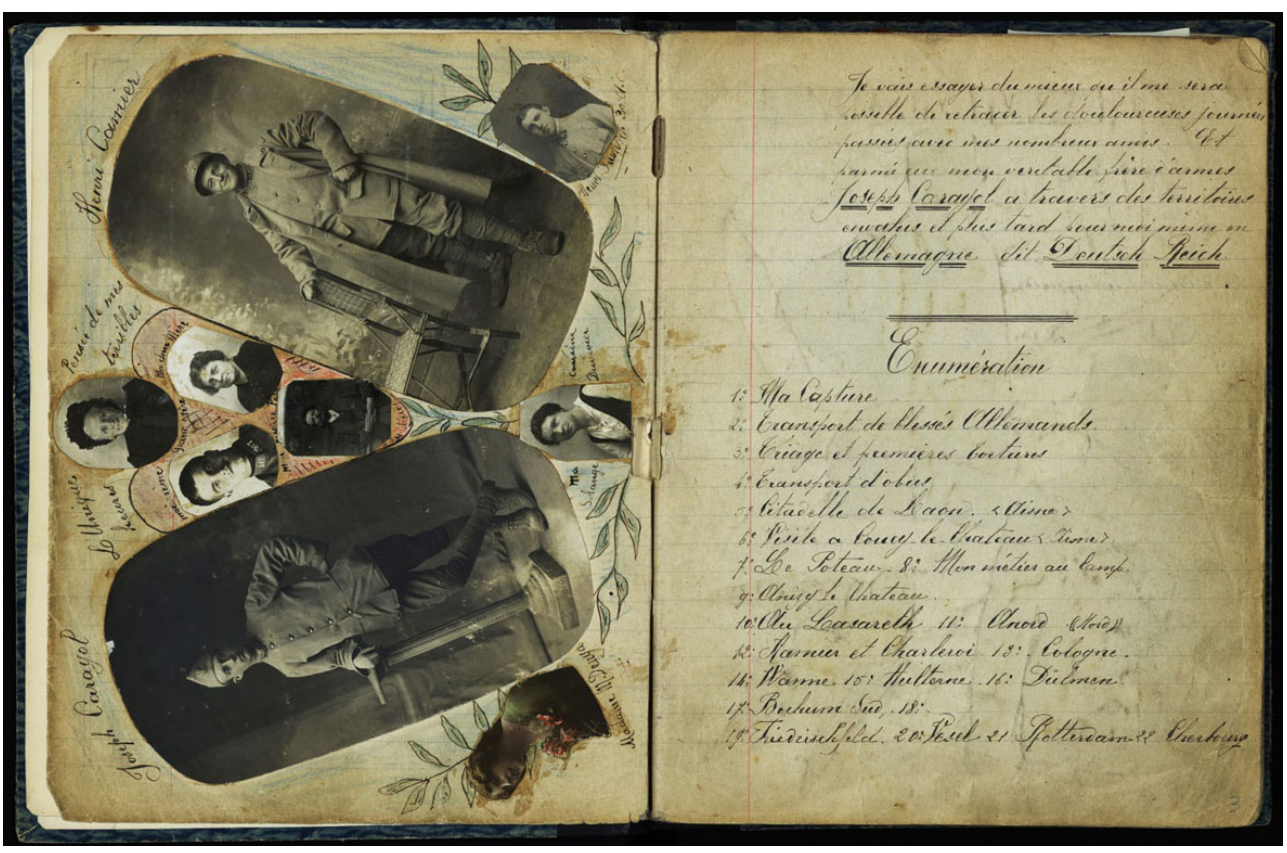

Carnet d'Henri Camier, prisonnier de guerre en Allemagne. AN, 700AP/PA_268.

(c) Archives nationales. 
Figure 23

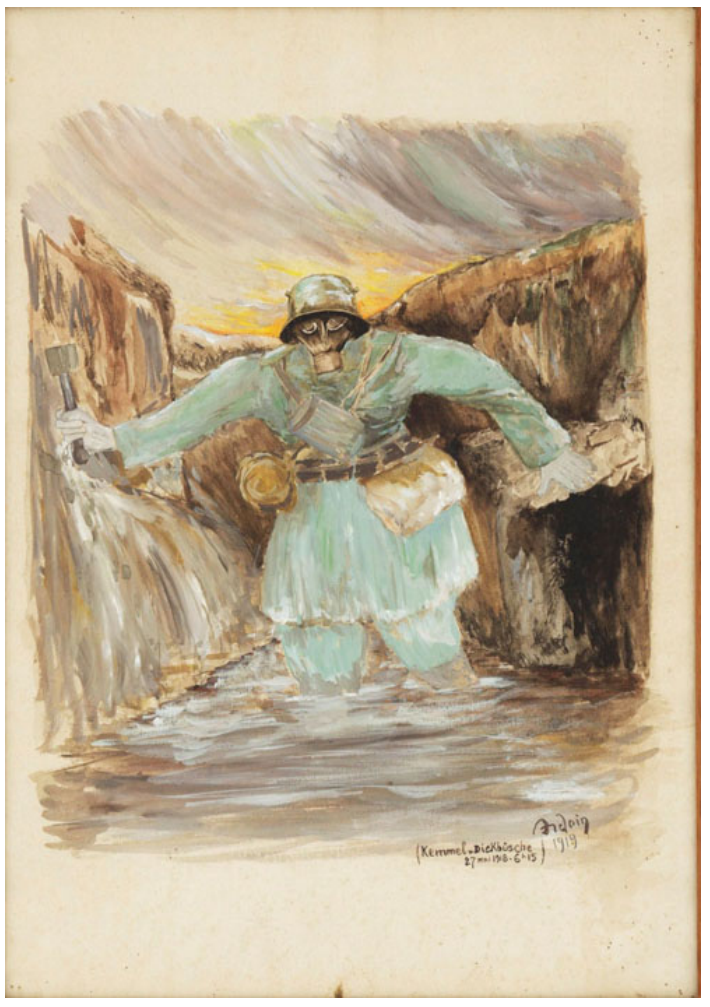

Dessin d'un soldat allemand dans les tranchées (1919), conservé par le soldat Gustave Ardoin. AN, 700AP/PA/346.

(C) Archives nationales. 
Figure 24

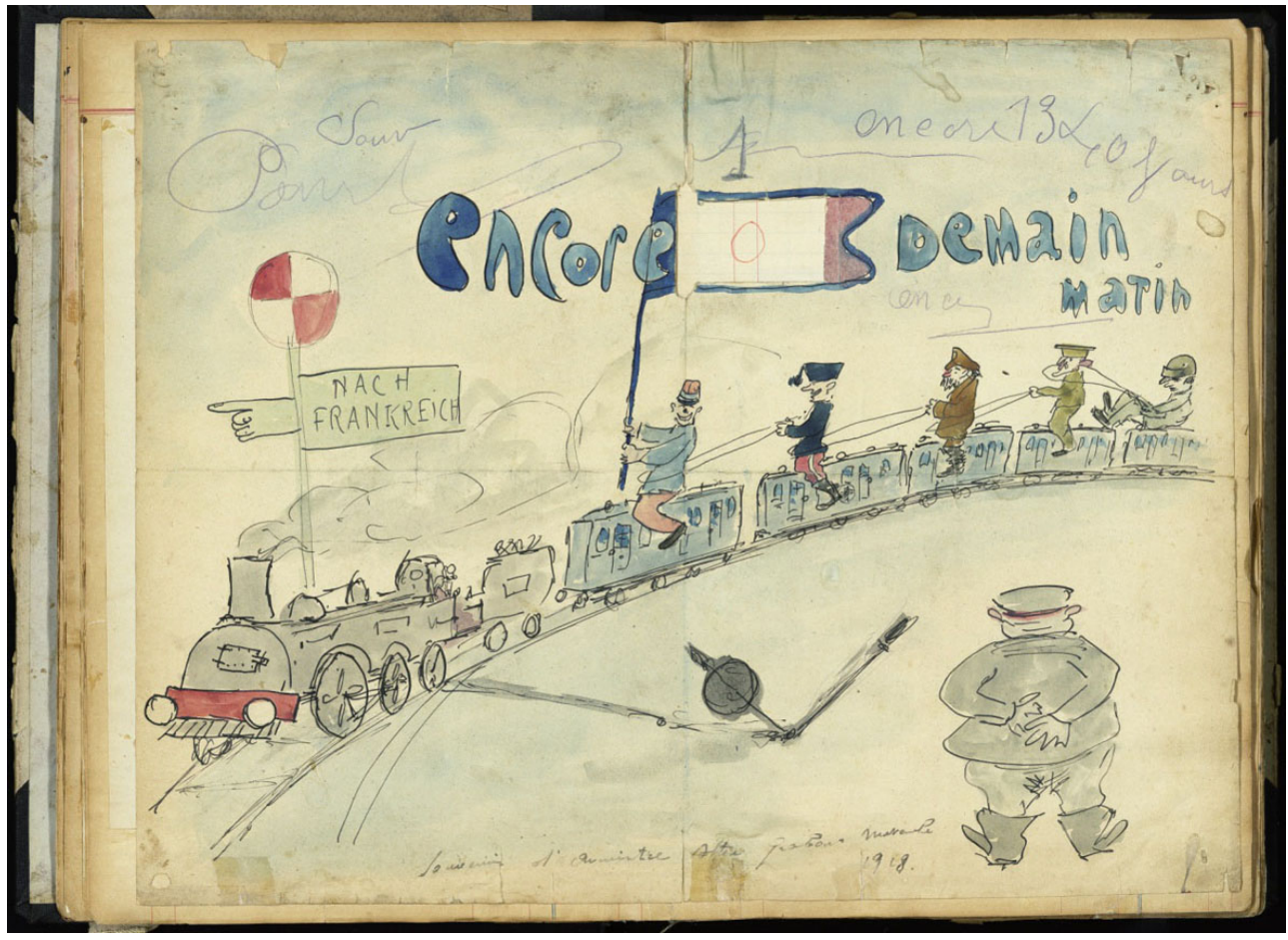

Dans le carnet du caporal Roger Fantoullier, la fin de la guerre vue avec humour. AN, 700AP/PA_023.

(c) Archives nationales.

Figure 25

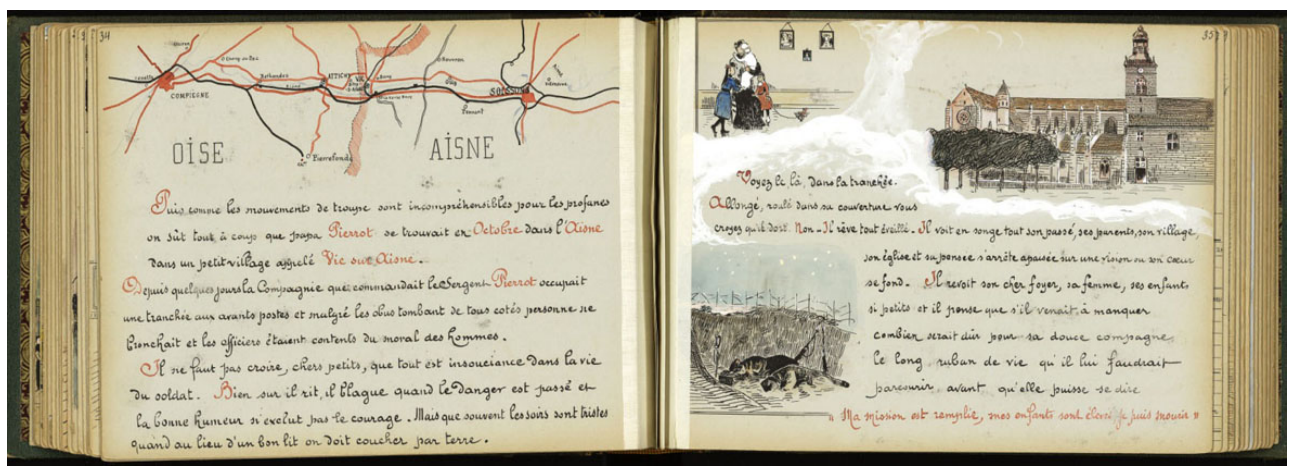

"Les deux familles. En souvenir du sergent Pierrot, mort pour la patrie », album manuscrit illustré par Henri Lecomte, 24e régiment d'infirmiers militaires. Fait exceptionnel, l'album d'Henri Lecomte est destiné à des enfants. AN, 700AP/PA_022.

(c) Archives nationales.

La Grande Collecte a apporté des milliers de cartes postales et de lettres échangées entre le soldat et ses proches. Dès le début de la guerre se met en place la correspondance militaire où l'adresse du soldat est remplacée par son secteur; mais beaucoup d'hommes envoient aussi des cartes photographiques les montrant en uniforme, des cartes représentant les ports traversés (pour ceux envoyés sur le front oriental), des vues de villages et d'églises détruits ou des cartes patriotiques. La plupart des correspondances ne contiennent que ces quelques mots « Je vais bien » répétés sous de multiples variantes, la carte ou la lettre étant davantage une preuve de vie qu'un témoignage. Mais malgré la censure, certaines lettres arrivent à décrire la réalité de la 
guerre. Paradoxalement, d'autres soldats n'enverront tout au long de la guerre que de délicates cartes ornées de dessins ou de rubans, bien loin de la réalité quotidiennement vécue. Les lettres écrites par les femmes ou les enfants de soldats, témoignages de la vie à l'arrière, ont été plus rarement conservées (fig. 26).

\section{Figure 26}

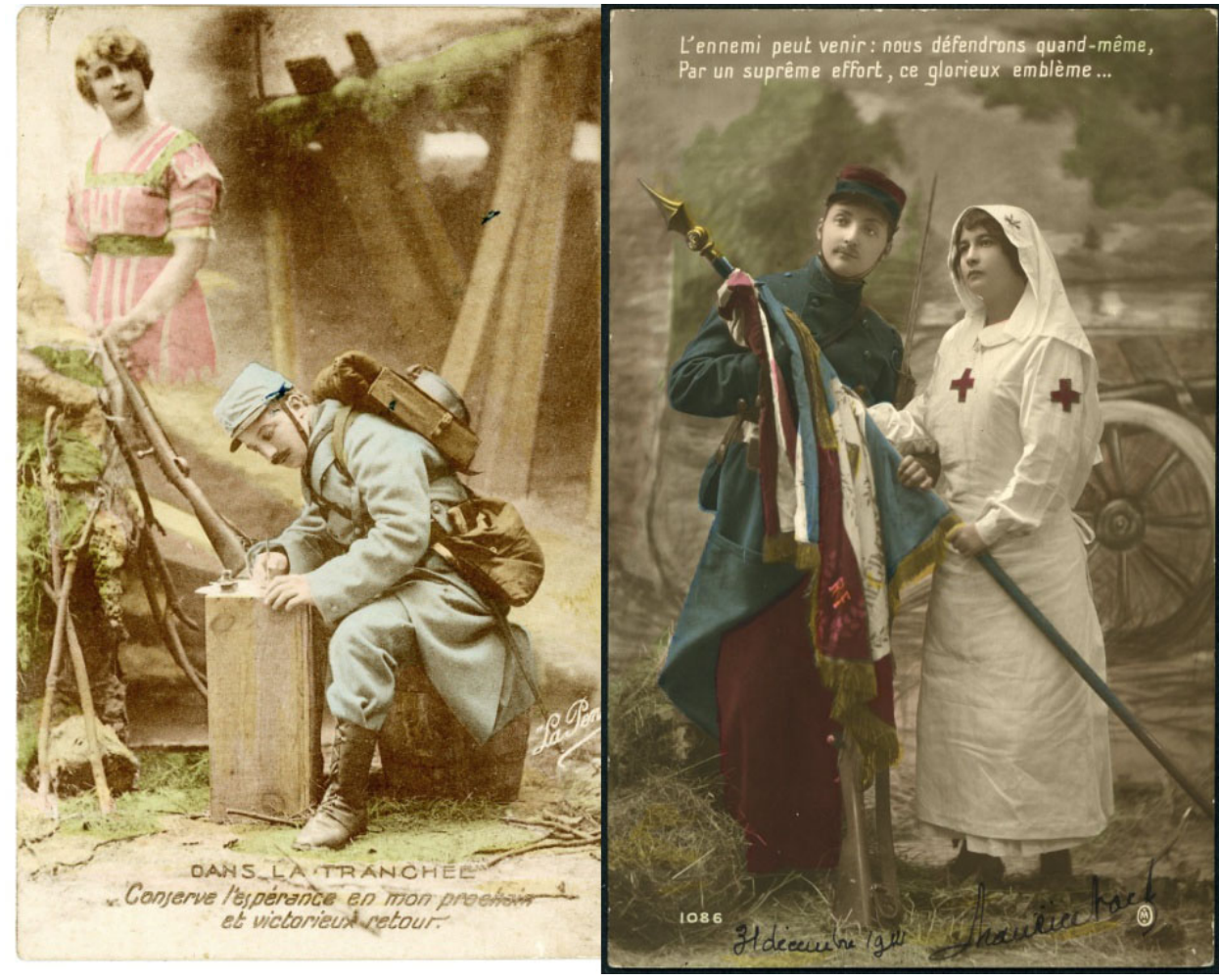

Cartes postales patriotiques : le soldat dans sa tranchée écrit à sa fiancée et carte de vœux envoyée par le soldat Émile Garret (31 décembre 1914). AN, 700AP et AN, 700AP/PA/231.

(c) Archives nationales.

Les cartes patriotiques exaltent les vertus guerrières et nationales, la haine du Boche, l'effort vers la victoire. La femme, souvent représentée en Jeanne d'Arc, sainte Geneviève ou en allégorie guerrière, est ici montrée comme participant à l'effort de guerre, soit comme infirmière soit comme soutien moral du soldat (fig. 27, 28, 29, 30). 


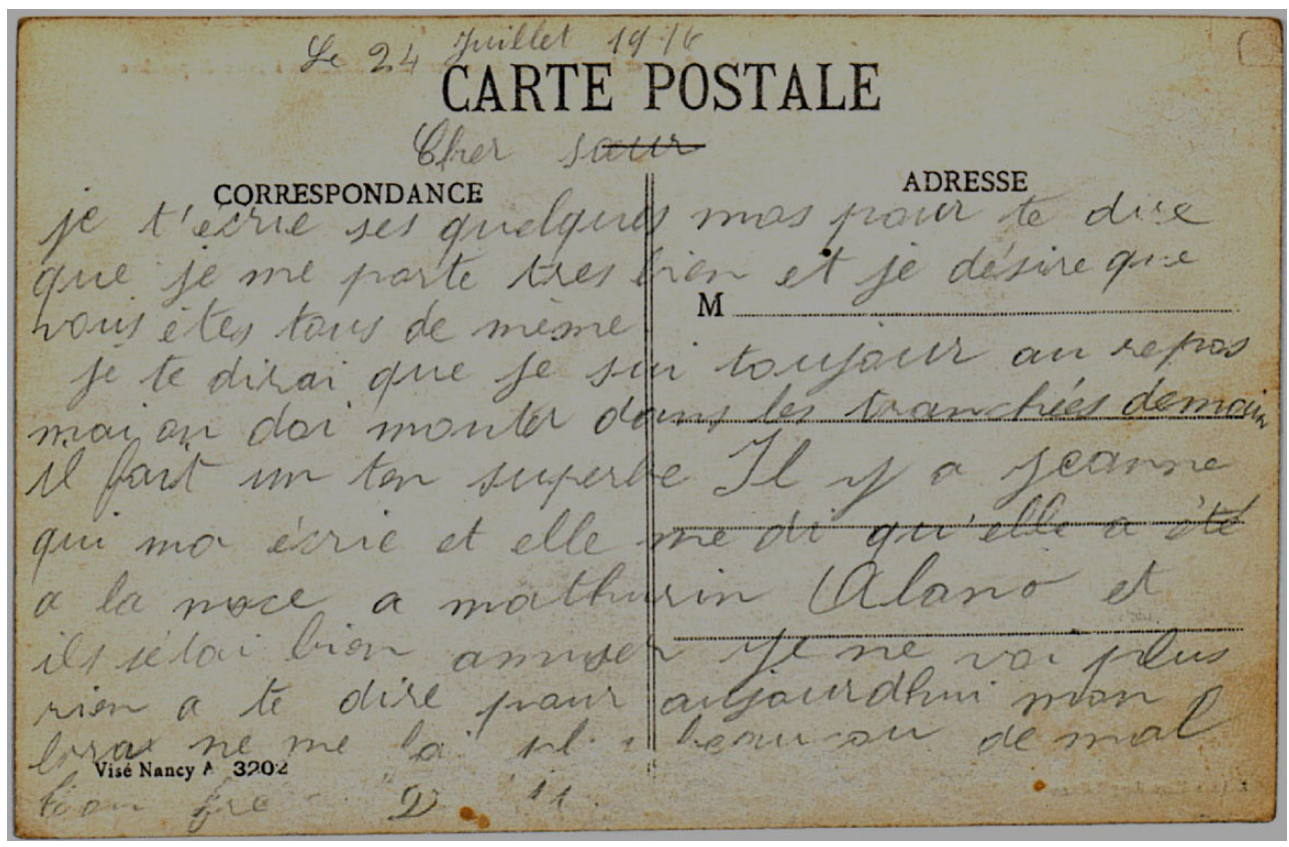

Carte du soldat Joseph Clément à sa sœur : « Je me porte très bien... » (24 juillet 1916). AN, 700AP/ PA_029.

(c) Archives nationales.

\section{Figure 28}

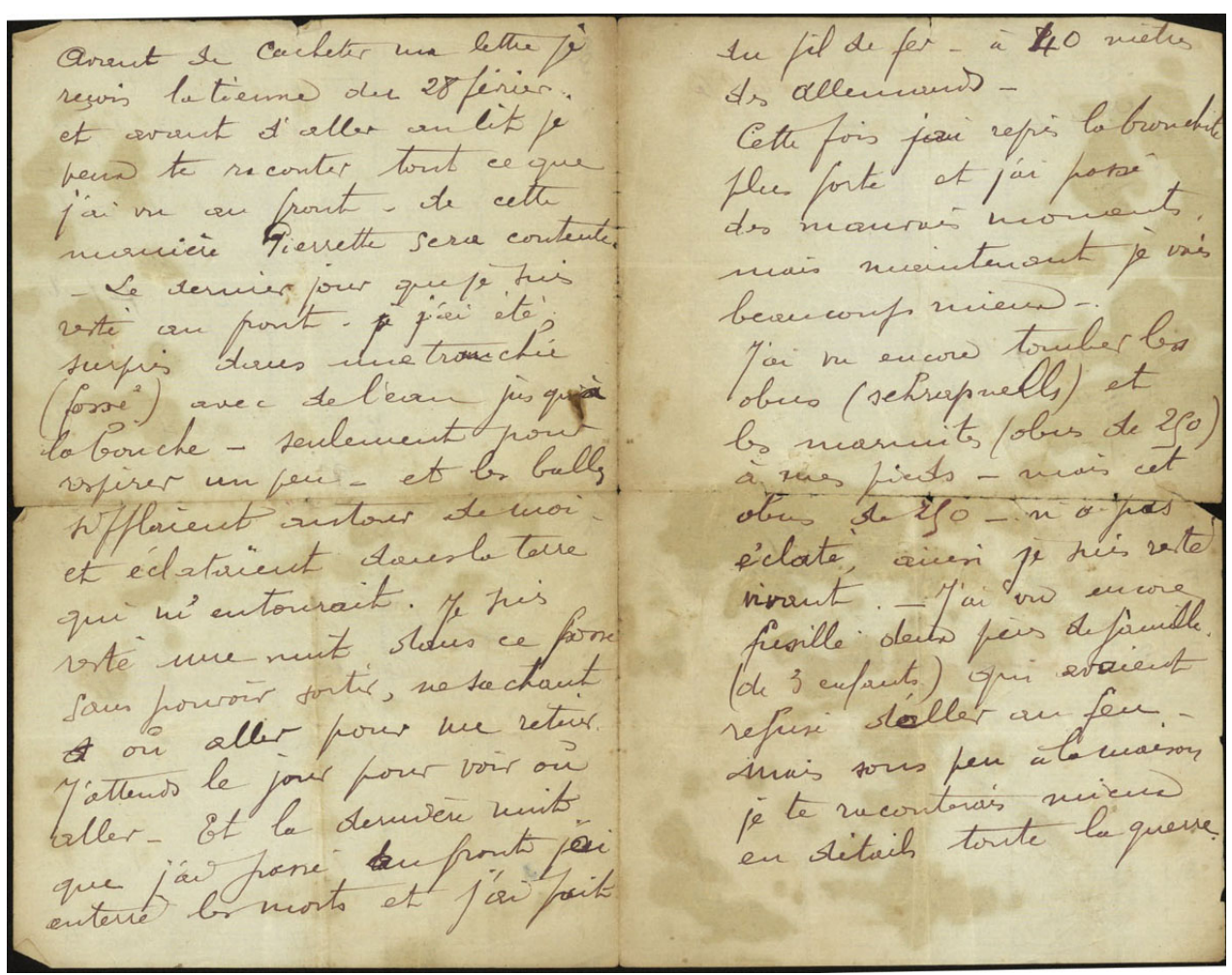

Lettre du soldat Ange Filippi à sa famille, décrivant les conditions réelles de la guerre : " J'ai été surpris dans une tranchée (fossé) avec de l'eau dans la bouche (...) et les balles sifflaient autour de moi... » (11 mars 1915). AN, 700AP/PA_099.

(c) Archives nationales. 


\section{Figure 29}

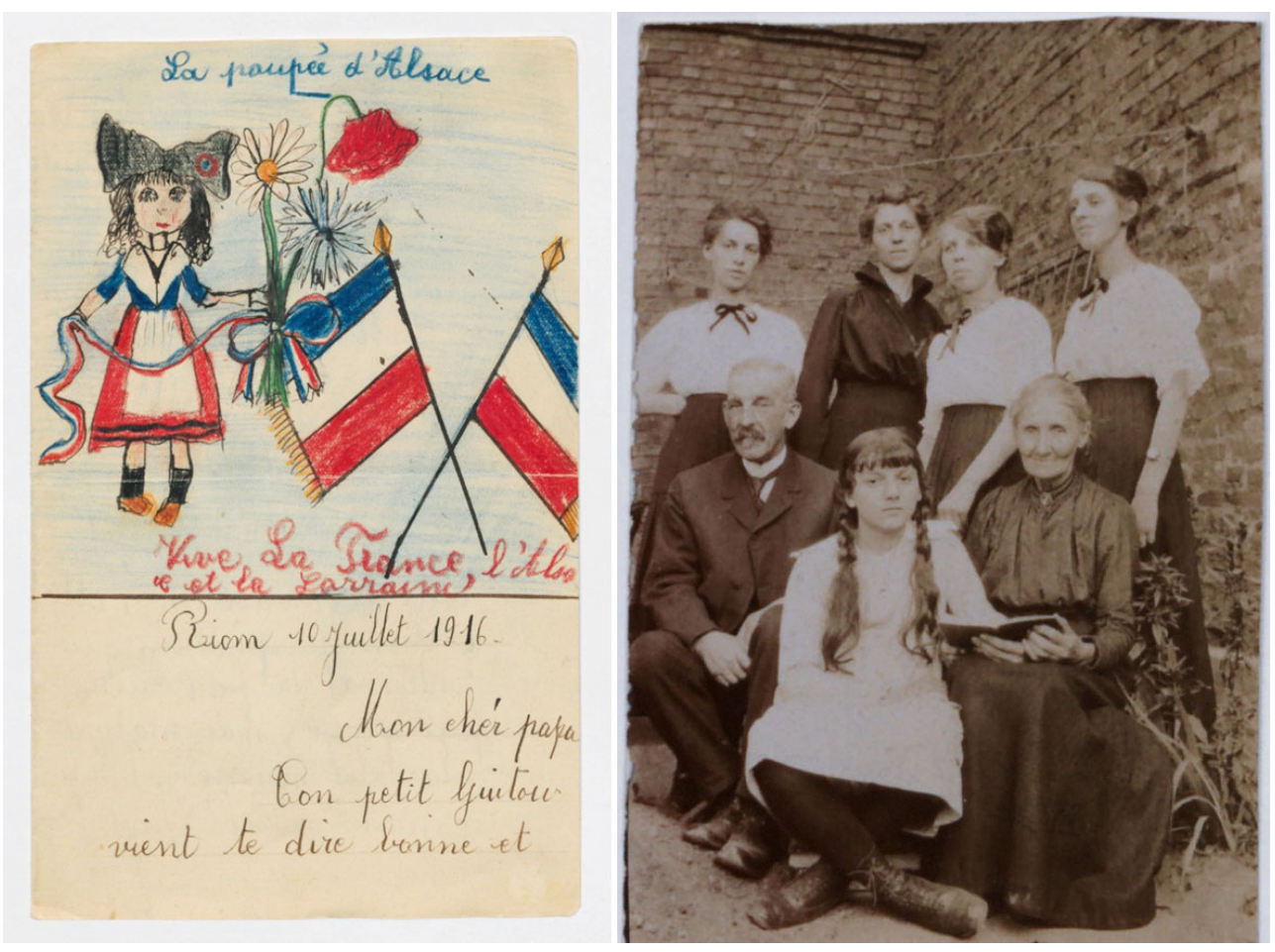

Lettre de Marguerite Hosmalin à son père (10 juillet 1916). Photographie de la famille Hosmalin pendant la guerre : une famille composée uniquement de femmes, d'enfants et de personnes âgées. La petite Marguerite, âgée de 8 ans en 1914, écrira à son père une centaine de lettres ornées de dessins. AN, 700AP/PA_101.

(C) Archives nationales. 


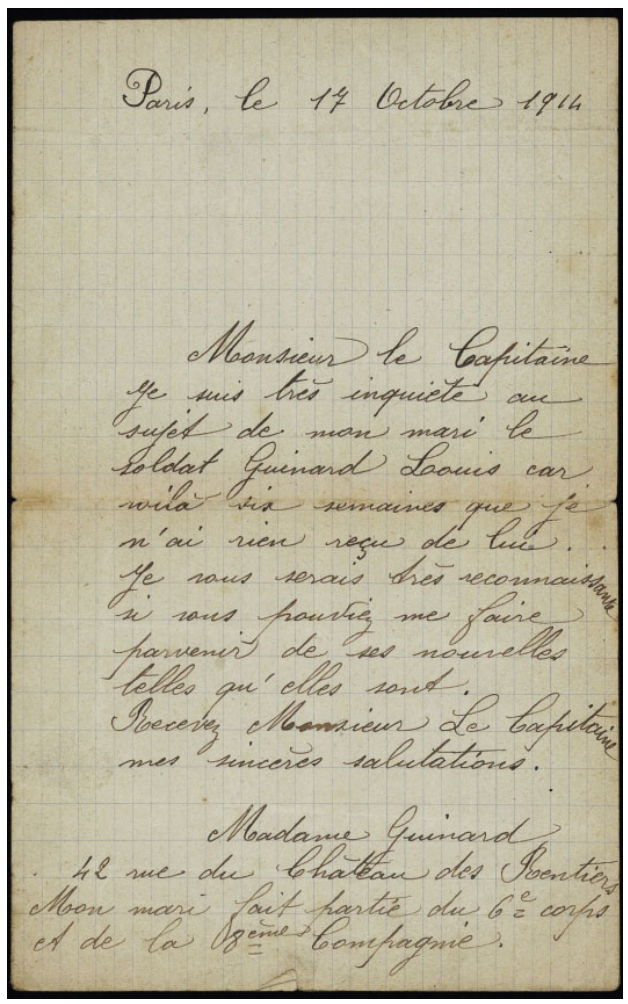

Lettre de Mme Guinard, s'inquiétant de la disparition de son mari. Dès le début, la guerre s'avère différente, très meurtrière. Les familles ne comprennent pas la disparition physique de leur proche déserteur, prisonnier de guerre, blessé ? Certaines épouses écriront pendant des années avant que leur mari ne soit déclaré « Mort pour la France ». AN, 700AP/PA_028.

(c) Archives nationales.

Comme pour les morts, l'image du soldat blessé représenté dans les documents de la Grande Collecte conservés par les familles est double: d'un côté, la photographie rassurante du soldat à l'hôpital, entouré d'infirmières et de médecins, des lettres touchantes témoignant des derniers instants du soldat; de l'autre des photographies réalistes, prises par des médecins, d'opérations chirurgicales, de gueules cassées, des carnets de notes d'infirmières ou des fiches sanitaires (fig. 31, 32). 
Figure 31

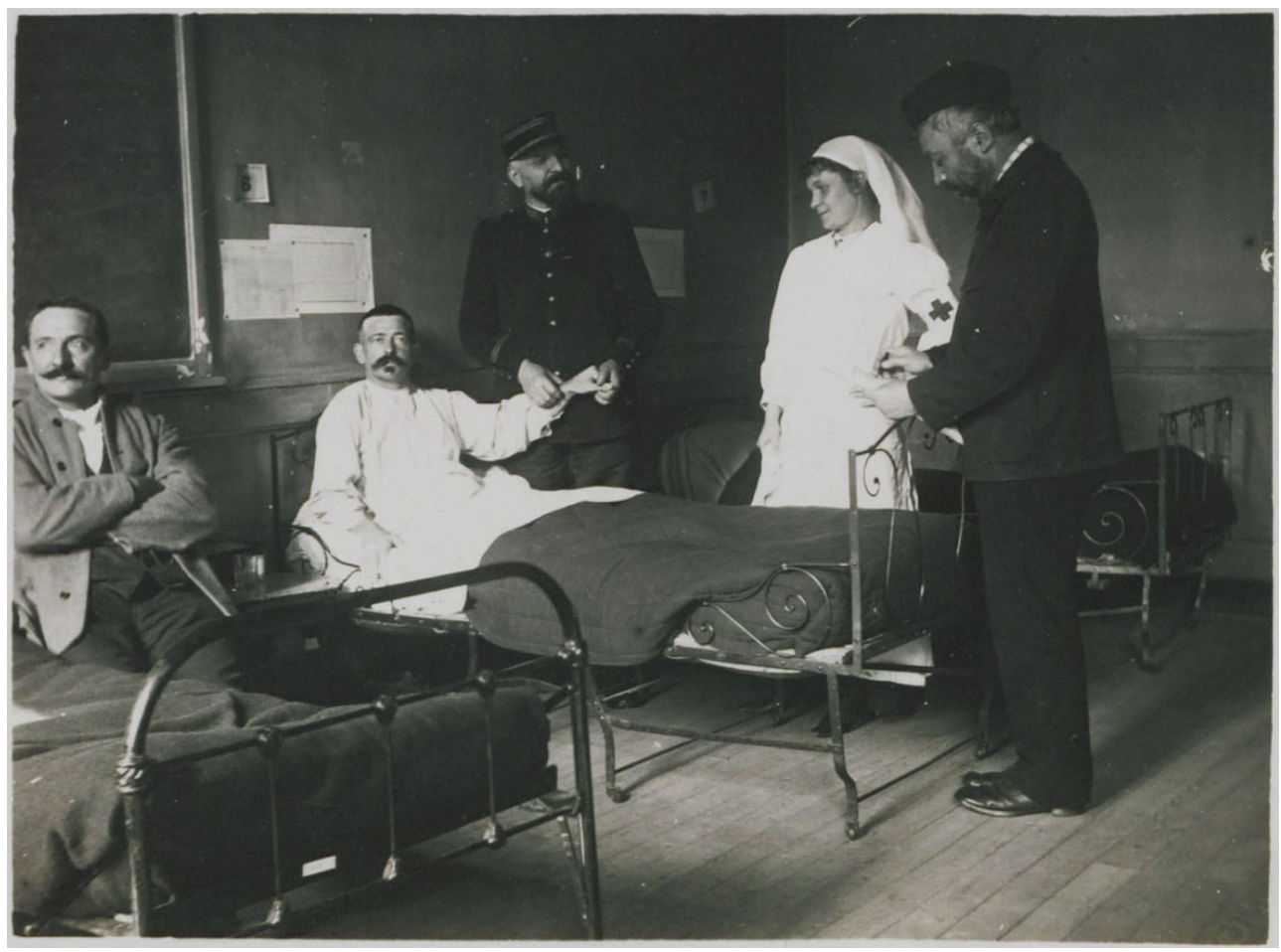

Le soldat Jean-Pierre Luminel à l'hôpital. AN, 700AP/PA_059.

(c) Archives nationales.

Figure 32

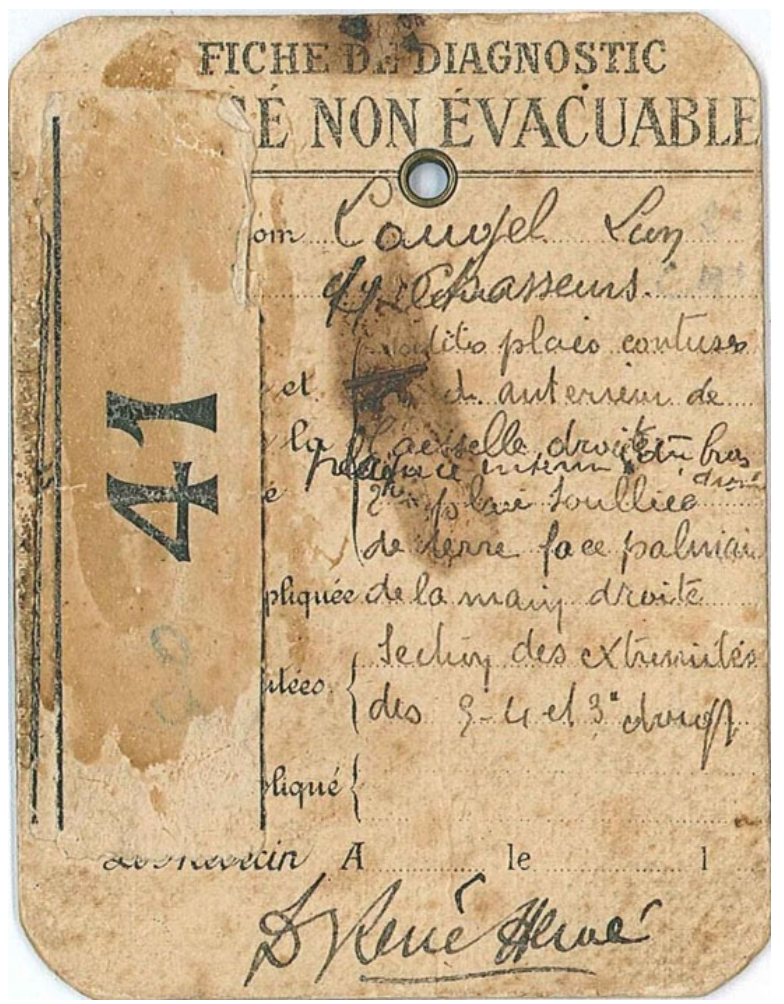

Fiche de blessé non évacuable au nom de Léon Louvel. AN, 700AP/PA_01.

(c) Archives nationales. 


\section{La valorisation du patrimoine privé de la Grande Guerre}

14 De nombreuses initiatives de valorisation locales, comme nationales, une série foisonnante de documentaires relayés dans les médias et mettant à profit ce type de sources - en particulier les journaux de guerre - dit bien l'intérêt que le public leur porte aujourd'hui. L'histoire au quotidien, vécue au plus près des populations - soldats, civils, femmes, enfants - s'en trouve éclairée sous un jour inédit.

Cet intérêt rencontre celui d'une association comme l'Association pour l'autobiographie, en la personne, notamment, d'un de ses fondateurs éminents, Philippe Lejeune, qui, après plusieurs autres livraisons du journal de l'association, $L a$ faute à Rousseau, réunit dans un numéro 67 consacré à la Grande Guerre ${ }^{1}$ une série d'articles dont chacun éclaire le profil d'un acteur, humble ou plus illustre. C'est l'occasion pour l'association de valoriser à la fois la part des ressources patrimoniales qu'elle conserve à Ambérieu-en-Bugey et des documents conservés dans des institutions publiques ou encore en mains privées, publiés ou inédits. Chercheurs en lettres, en histoire, écrivains rendent compte, chacun à leur manière, de l'apport renouvelé de ces sources pour la compréhension de la Grande Guerre, des deux côtés du Rhin.

L'occasion nous est donnée enfin d'annoncer (voir notre annexe) la tenue, les 22 et 23 janvier prochains aux Archives nationales et à la fondation Singer-Polignac à Paris d'un colloque Écrire en guerre (1914-1918). Archives privées, usages publics. Coorganisée par la Fondation, les Archives nationales et la société des Amis des Archives de France, cette manifestation réunira de nombreux acteurs œuvrant, aujourd'hui, à la collecte et à la valorisation patrimoniale et scientifique de ce for privé de la Grande Guerre dont les ressources, pour les chercheurs comme pour le grand public, sont loin d'être épuisées.

\section{APPENDIXES}

\section{Programme du colloque international}

Archives nationales Société des amis des archives de France

Fondation Singer-Polignac

Écrire en guerre

Archives privées, usages publics

22-23 janvier 2015

22 janvier 2015

Lieu : Archives nationales, Pierrefitte-sur-Seine, auditorium 
(59, rue Guynemer 93280 Pierrefitte-sur-Seine, métro Saint-Denis Université)

9h. Accueil.

9h30. Ouverture du colloque par Mme Françoise Banat-Berger, directrice des Archives nationales et par $\mathrm{M}^{\mathrm{e}}$ Alain Moreau, président de la société des amis des Archives de France.

10h-12h30. $1^{\mathrm{e}}$ séance : Les organismes acteurs

Président : Antoine Prost

10h. « La Grande collecte 14-18 : retour sur un projet mémoriel interactif », par Emmanuel Pénicaut (service interministériel des Archives de France) et Isabelle Aristide-Hastir (Archives nationales).

10h30-12h30. Table ronde : « Les organismes acteurs : caractéristiques, complémentarité ».

Modérateur : Catherine Dhérent (Métropole européenne de Lille).

Participants : Isabelle Aristide; Françoise Aujogue (ministère des Affaires étrangères); Agnès Chablat-Beylot (service historique de la Défense) ; Marlene Kayen (Deutsche Tagebucharchiv, Emmendingen, Allemagne); Claudine Krishnan (Association pour l'autobiographie) ; Emmanuel Pénicaut (service interministériel des Archives de France) ; Olivier de Solan (archives départementales) ; Valérie Tesnière (bibliothèque de Documentation internationale contemporaine) ; Marie-Pascale Prévost-Bault (Historial de la Grande Guerre à Péronne) ; Bruno Vouters (La Voix du Nord).

12h30. Déjeuner, avec possibilité de visite libre de l'exposition « Août 14. Tous en guerre!»

13h45. Concert lecture par l'ensemble TM+.

14h30-17h30. $2^{\mathrm{e}}$ séance : Qui écrit ? Qu'écrit-on?

Président : Jay Winter

14h30. François-Joseph Ruggiu (Paris IV) : Typologie du for privé.

15h. Odile Gaultier-Voituriez (Sciences-Po, Cevipof) : La guerre vue de Paris par Étienne de Nalèche, directeur du Journal des Débats.

15h30. Hubert Roland (F.R.S.-FNRS, université catholique de Louvain) : Les journaux de Thea Sternheim. For privé, socialisation et politique.

16h. Fabien Théofilakis (université de Montréal) : De l'écriture en captivité à l'écriture captive : quand les prisonniers écrivent...

16h30. Ludmila Evdokimova (Institut de littérature mondiale, Moscou) : Journal de Sophia Ilovaïskaïa : les épisodes de guerre (1915-1917).

17h. Débat

17h30. Visite guidée (sur inscription) de l'exposition Août 14. Tous en guerre !, par Isabelle Chave, commissaire de l'exposition.

\section{3 janvier 2015}

Lieu : fondation Singer-Polignac 
(43, avenue Georges-Mandel 75116 Paris)

\section{9h30-12h30. $3^{\mathrm{e}}$ séance : Témoins engagés}

Président : Jean-Noël Jeanneney

9h30. Clotilde Druelle-Korn (université de Limoges) : Surveiller le ravitaillement de la Belgique et de la France occupée : les témoignages des premiers délégués américains de la Commission for Relief in Belgium.

10h. Marie Scot (Centre d'histoire de Sciences-Po) : Écrire ou ne pas écrire en guerre : le choix d'Élie Halévy.

10h30. Philippe Verheyde (université de Paris 8) : Journaux du préfet Trépont, une chronique engagée de la guerre à la déportation (1914-1915).

11h. Pause-café

11h15. Zénaïde Romaneix (Archives nationales) : La correspondance reçue par les députés français pendant la guerre.

11h45. Romain Ducoulombier (Centre d'histoire de Sciences Po) : Henri Barbusse. Écriture, réécriture et réception d'une expérience de guerre.

12h15. Débat

12h30-14h. Déjeuner-buffet

14h-17h. $4^{\mathrm{e}}$ séance : Usages et résonance

Président : Philippe Lejeune

14h. Philippe Lejeune (université Paris 13 et APA) : Nous autres à Vauquois (1918 d'André Pézard : l'après-coup de la guerre.

14h30. Benjamin Gilles (BDIC) : Interroger les anciens combattants : Norton Cru et la préparation de Témoins.

15h. Philippe Henwood (Inspection générale des patrimoines) : Si Dame Camarde a le bon goût de m'épargner...

15h30. Rémy Cazals (université de Toulouse Jean-Jaurès) : Louis Barthas et la postérité.

16h. Débat.

16h30. Conclusions par Paule René-Bazin et Elsa Marguin-Hamon.

\section{NOTES}

1. - Voir le site : http://autobiographie.sitapa.org/publications/faute-a-rousseau/sommaire.php. 


\section{ABSTRACTS}

The operation called 'La Grande Collecte', the great collection, is an initiative of the French national library aimed at collecting and copying private documents relating to the First World War. This operation was carried out from 9 to 16 November 2013 and met with considerable success both at the national archives and throughout the network of fifty libraries which participated in the operation. Private individuals were invited to bring family documents relating to the First World War, documents which could be digitised and published on line on the site Europeana Collections 1914-1918, along with their own account of the origins of the papers. The documents are therefore conserved only in digital form, although many donors in the end preferred to give the originals to the archive services, aware that the papers are now fragile and that their conservation within the family was perhaps not guaranteed. The harvest, at first sight, is both diversified and repetitive: letters, post cards, photographic portraits of soldiers, military service record books, private notebooks, drawings and so on. But taken as a whole and forming part of a shared heritage of memory associated with exceptional events (perceived as such by the witnesses), the collection now forms a national treasure without equivalent at the national archives.

Menée à l'initiative de la Bibliothèque nationale de France, l'opération Grande Collecte 1914-1918, qui s'est déroulée en France du 9 au 16 novembre 2013, a connu un large succès public tant dans la cinquantaine de sites d'accueil du réseau des bibliothèques et des archives en France qu'aux Archives nationales. Les particuliers étaient invités à apporter, pour numérisation, des documents de la guerre 14-18, qui seront publiés, avec leur témoignage, sur le site « Europeana Collections 1914-1918». Les documents sont donc conservés essentiellement sous forme numérique, mais de nombreux particuliers, conscients de la fragilité des supports et de l'incertitude de leur conservation au sein de leur famille, ont consenti à un don immédiat aux Archives nationales, ou dans les années à venir. L'ensemble des apports est, de prime abord, hétéroclite ou récurrent (lettres, cartes postales, photographies de soldats, livrets militaires, carnets de notes de poilus, citations, dessins) ; mais, s'inscrivant dans un patrimoine mémoriel commun, associé à un événement exceptionnel et ressenti comme tel par les contemporains, il a le caractère d'un trésor patrimonial, unique en son genre aux Archives nationales.

\section{INDEX}

Keywords: national archives, private archives, Grande collecte, great collection, First World War, private writings, digitisation, written heritage

Mots-clés: Archives nationales, archives privées, Grande Collecte, guerre de 1914-18, for privé, numérisation, patrimoine écrit

\section{AUTHORS}

\section{ISABELLE ARISTIDE-HASTIR}

Conservateur en chef du patrimoine, responsable du département des Archives privées, département des Archives privées, Archives nationales isabelle.aristide@culture.gouv.fr 


\section{ELSA MARGUIN-HAMON}

Conservatrice du patrimoine, Archives nationales, Direction des publics, Département de l'action culturelle et éducative Musée des Archives nationales elsa.marguin@culture.gouv.fr 